\title{
Mujer y partidos políticos en España: las estrategias de los partidos y su impacto institucional, 1978-2004
}

Women and political parties in Spain: party strategies and their institutional impact, 1978-2004

Palabras clave: Partidos Políticos, Representación Política, Discriminación Positiva, Cuota.

RESUMEN

El presente artículo realiza un análisis en profundidad de la respuesta de las tres formaciones políticas de ámbito estatal (Partido Socialista, Partido Popular e Izquierda Unida) a la reivindicación de una presencia equilibrada de mujeres y hombres en los puestos de responsabilidad política; una demanda que atañe a los partidos en tanto que entre sus funciones operativas se encuentra el reclutamiento de las elites políticas, y es esta selección de candidatos la que determina finalmente la configuración de las instituciones políticas. En concreto, analiza las estrategias implementadas por los tres partidos en el período democrático iniciado en 1978 y estudia su efecto sobre la presencia de las mujeres en los cargos de máxima responsabilidad orgánica y en las instituciones representativas. Se constata que el incremento en la representación femenina se ha producido tanto por cuestiones ambientales (la competición electoral entre los partidos, la lucha por el voto femenino, etc.)
Keywords: Political Parties, Political Representation, Affirmative Action, Quota.

\section{ABSTRACT}

This article examines in depth the answer that the three main state-wide Spanish political parties (Spanish Socialist Workers' Party, Popular Party and United Left) have given to the demand for a gender balanced presence in representative offices; a demand which greatly concerns political parties as, among their operative functions, they are in charge of the recruitment of political elites and candidate selection which finally determines the configuration of political institutions. In particular, it analyzes the strategies implemented by political parties since the inauguration of democracy in 1978 and studies their effects on the presence of women holding top posts in internal party bodies and representative institutions. The increase in women's representation has been due to exogenous factors (electoral rivalry among parties, competition for women's vote, etc.) as well as to internal reasons such as the political 
como por cuestiones internas, como la ideología política del partido, la constitución de lobbies de militantes feministas en los partidos y la introducción de cuotas. ideology of the party, the creation of feminist lobbies inside the parties and the introduction of quotas. 


\section{INTRODUCCIÓN}

En este artículo se estudia el efecto de las estrategias implementadas por los tres partidos de ámbito estatal sobre la presencia de las mujeres en los cargos de máxima responsabilidad orgánica y en las instituciones representativas. Para ello realizaremos un análisis en profundidad de la respuesta del Partido Socialista, del Partido Popular y de Izquierda Unida a la reivindicación de una presencia equilibrada de mujeres y hombres en los puestos de responsabilidad política; una demanda que atañe a los partidos en tanto que entre sus funciones operativas se encuentra el reclutamiento de las elites políticas, y es esta selección de candidatos la que determina finalmente la configuración de las instituciones políticas. Es decir, la responsabilidad última de que un Parlamento contenga un porcentaje determinado de mujeres reside en última instancia en los partidos, más allá de las características del sistema electoral vigente.

En este recorrido por las estrategias implementadas por los tres partidos distinguiremos entre estrategias retóricas, de acción positiva y de discriminación positiva. En primer lugar, son estrategias retóricas aquellas que suponen la difusión de esta demanda en los documentos de los partidos y las referencias de sus dirigentes a la importancia de la incorporación de las mujeres en la vida política. En segundo lugar, los partidos pueden aplicar estrategias de acción positiva, que incluyen programas de formación para afiliadas y/o candidatas, la creación de secciones específicas de mujeres y el establecimiento de relaciones con organizaciones feministas. Y, por último, las estrategias de discriminación positiva se materializan en la reserva de puestos en los órganos ejecutivos del partido o en las listas electorales ${ }^{1}$ (Lovenduski y Norris, 1993).

En el primer apartado del artículo analizaremos las estrategias implementadas por los tres partidos en el período democrático iniciado en 1978. En segundo lugar, estudiaremos el diverso efecto que los tres tipos de estrategias han tenido sobre la presencia de las mujeres en los cargos de máxima responsabilidad orgánica. Posteriormente, se analizará la evolución de la participación de las mujeres en las instituciones para contrastar el impacto de las medidas implementadas. En ambos casos prestaremos especial atención a las motivaciones y factores que han inducido a los partidos a incrementar la representación femenina. Por último, se reflexionará sobre las limitaciones de la cuota y sobre los mecanismos más sutiles que se intrincan en la aplicación de la discriminación positiva y que matizan su impacto.

\footnotetext{
1 Aunque, generalmente, se identifican las expresiones «acción positiva» y «discriminación positiva», entendemos que tienen un matiz importante que permite presentarlas por separado: mientras que las primeras consisten en actuaciones destinadas a favorecer a un grupo determinado sin perjudicar directamente a nadie, las segundas sí tienen consecuencias para otros grupos. En este caso, la cuota supone conceder una reserva de puestos a un grupo en detrimento del «cupo» del que disfruta otro grupo.
} 


\section{LAS ESTRATEGIAS DE LOS PARTIDOS}

\section{Estrategias retóricas}

Para conocer la importancia otorgada a la presencia de la mujer en la vida política se ha realizado una revisión sistemática de las resoluciones congresuales y de los programas electorales presentados por los partidos. Los programas electorales, en tanto que declaración pública de las políticas que el partido quiere implementar, han sido utilizados como una «definición autorizativa» de la posición de un partido, del mínimo ideológico que lo define en cada momento (Budge et al., 2001). Otra fuente de valiosa información son las resoluciones aprobadas en los congresos de los partidos, ya que fijan las líneas prioritarias de acción y de organización. También se han utilizado declaraciones de dirigentes de los partidos aparecidas en los medios de comunicación.

En lo que a las estrategias retóricas se refiere, las diferencias halladas entre los tres partidos son destacables. Durante los primeros años ochenta, tras la incorporación de algunas mujeres a los órganos de dirección del PSOE y del PCE (y posteriormente de IU), los temas «de mujer» fueron redefinidos como demandas por la igualdad entre géneros: las mujeres no eran un sector, sino la mitad de la población. A pesar de que en esos años la agenda política de la igualdad estaba dominada por propuestas relativas al mercado laboral, la educación o las políticas sociales, la representación de las mujeres se transformó paulatinamente en una demanda que pretendía mejorar la calidad de la democracia: siendo las mujeres la mitad de la población, les correspondía de forma legítima una participación mucho mayor en los puestos de responsabilidad de la que gozaban hasta la fecha (PSOE, 1988: 18). En esta línea, el programa electoral de IU presentado en 1986 manifestaba: «Las mujeres, que representan el $52 \%$ de la población, son olvidadas y marginadas, tanto en su representación política y parlamentaria como en su situación económica y social» (IU, 1986: 27).

Posteriormente, al presentar los partidos de izquierda la infrarrepresentación de las mujeres como un «déficit democrático» (PSOE, 1993: 60), resultaba difícil exponer argumentos en contra de su demanda: «Una sociedad democrática no puede permitir la exclusión o la marginalidad política de las mujeres cuando su sistema está basado en la igual soberanía de todas las personas. El desarrollo de la democracia, como el avance hacia la igualdad social, no se entiende sin la presencia de las mujeres y los valores que éstas aportan. Sin las mujeres no es posible un desarrollo en profundidad de la democracia, porque sería prescindir de la participación de la mitad del género humano» (PSOE, 1994: 91). De este modo, IU y el PSOE han conferido a la representación paritaria un alto contenido simbólico basado en el argumento de la justicia (si las mujeres son la mitad de la población, es justo que ocupen la mitad de los puestos políticos), luchando por romper la percepción subjetiva 
de que la política es cosa de hombres: «La experiencia positiva del aumento de la presencia femenina en la última década contribuye a sustentar la idea de que las mujeres mejoran la cultura y la vida política, hasta el punto de que su aportación es esencial para hacer frente a los grandes retos del mundo actual» (IU, 2004a: 24).

En los programas electorales este apoyo discursivo se ha traducido en propuestas para fomentar el asociacionismo femenino y para conseguir una participación paritaria en los órganos representativos y de toma de decisiones en los ámbitos políticos, sociales y culturales, incluyendo la reforma del procedimiento administrativo de contratación funcionarial, la reforma de la Ley Electoral para garantizar una presencia equilibrada en las listas, la configuración de gobiernos paritarios o la eliminación de la discriminación sobre la mujer en el acceso a la Jefatura del Estado (Verge, 2005: cap. 4).

Por el contrario, no será hasta 1993 cuando el PP se manifestará a favor de la ampliación de la participación de las mujeres en la vida económica y social (PP, 1993: 21). Unos meses antes de las elecciones generales de 1996, el partido declaraba que la plena igualdad de la mujer en la sociedad española era un «objetivo irrenunciable» del partido, pero insistía en el siguiente posicionamiento: «No se trata de establecer unas discriminaciones legales positivas que, además de contravenir la legislación europea, pueden ser contraproducentes» (PP, 1996: 66). Este mayor énfasis en la participación de la mujer no se trasladará a los programas electorales presentados en años posteriores, reapareciendo de forma muy vaga en el programa electoral de 2004 (PP, 2004a: 109).

\section{Estrategias de acción positiva}

En este caso, se ha analizado la estructura organizativa de los tres partidos y las actividades formativas realizadas en el período 1978-2004 cuyo objetivo fuese la igualdad en la representación política.

En el PSOE e IU la preocupación por incrementar la militancia femenina ha sido una constante en todo el período estudiado, aunque esta voluntad no se ha materializado siempre en campañas concretas. EI PSOE implementó en 1990 la campaña «Cada una, una» y hasta 1997 no se plantearon nuevas campañas de afiliación femenina como estrategia permanente entre congresos. En IU, a pesar de la insistencia del partido en la necesidad de incrementar la afiliación femenina, las campañas no han sido muy frecuentes y no se ha implementado ninguna específica para mujeres. Los limitados recursos económicos con los que ha contado la organización han sido un pesado constreñimiento para ello². Paradó-

\footnotetext{
2 Maite Mola, ex responsable del Área de Mujer de IU y Secretaria de Mujer del PCE, entrevista personal, 7/2/2005.
} 
jicamente, como se puede observar en el gráfico 1, el PP es el partido que más afiliadas tiene sin haber emprendido nunca campañas de afiliación destinadas a mujeres.

GRÁFICO 1

Ratio Mujeres afiliadas/Total afiliación (M/T)

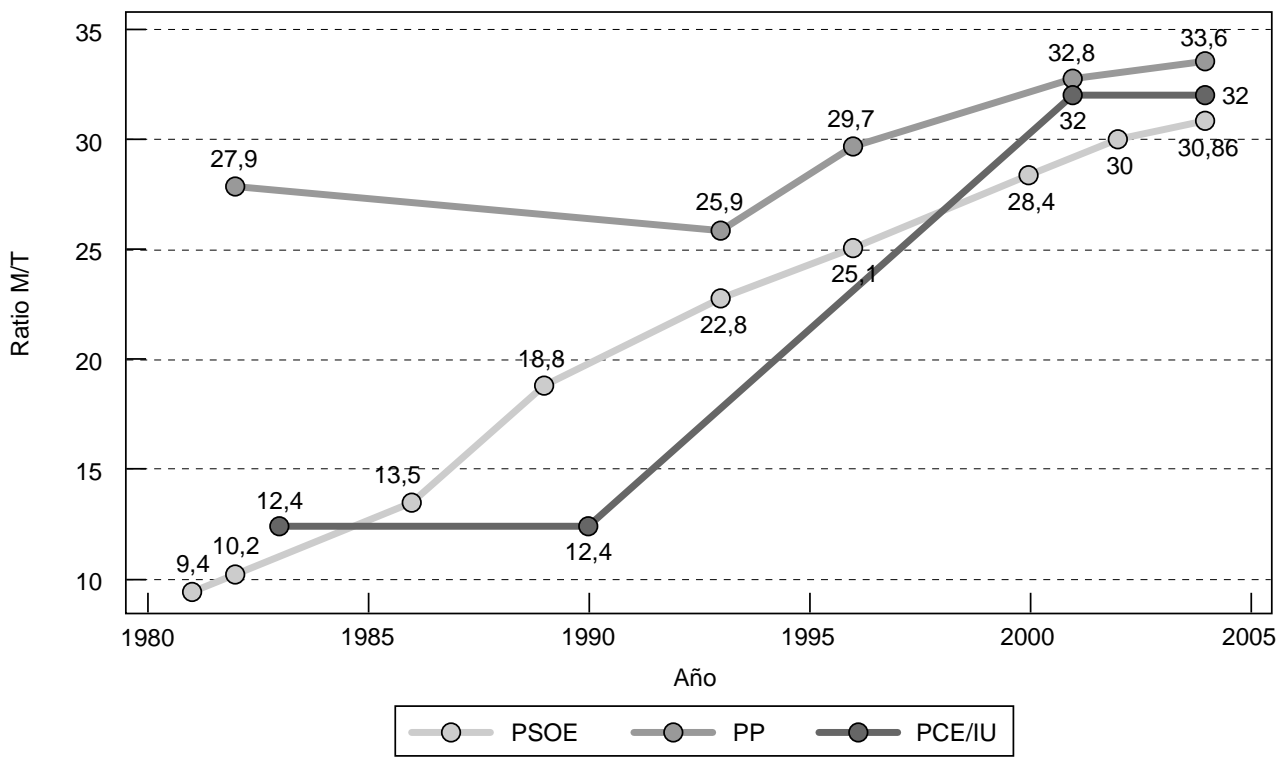

FUENTE:

Elaboración propia. Datos del PSOE: PSOE (2001) y datos facilitados por la Secretaría de Organización del PSOE. Datos de IU: Ramiro (2004) e Instituto de la Mujer (1999). Datos del PP: Sánchez Férriz (2000: 232) y PP (2002 y 2004b).

Entre las razones que pueden explicar una mayor militancia femenina en los partidos de derecha, se ha señalado que las mujeres de estos partidos suelen proceder en mayor medida de clases acomodadas, lo que les permitiría liberarse con más facilidad del trabajo doméstico. Perviviría también en estas mujeres una cultura tradicional con un claro sesgo hacia la derecha (García Escribano y Frutos, 1999: 316). No hay que olvidar que los partidos de izquierda se han desarrollado en estrecha colaboración con los sindicatos y el mundo del trabajo, ámbito en el que la mujer se ha incorporado mucho más tarde que el hombre, y donde todavía hoy su tasa de actividad es mucho menor a la de los hombres. Por otra parte, muchos partidos de la derecha han mantenido estrechas relaciones con la Iglesia, institución a la que las mujeres estuvieron tradicionalmente vinculadas y en la que muchas de 
ellas iniciaron sus actividades públicas (Uriarte, 1995: 126). La incorporación paulatina de la mujer al mercado laboral y la erosión de la influencia de la Iglesia habrían debilitado la ventaja de los partidos de derecha. En el año 2004, la afiliación era del 33,6\% en el PP, $32 \%$ en IU y $31 \%$ en el PSOE. Sin embargo, de 1980 a 2004 la tasa de crecimiento de la afiliación femenina en el PP ha sido tres veces inferior a la de los otros dos partidos.

Desde el PSOE e IU también se ha dado mucha importancia a la formación política de las afiliadas, aunque sólo el primero de los partidos ha realizado de forma sistemática desde finales de los años setenta diferentes ciclos formativos. Con esta formación se pretendía tanto evitar que el mayor reclutamiento de mujeres fuera cuestionado por ciertos sectores del partido debido a la menor educación política de las militantes como elevar la autoestima de las propias afiliadas $^{3}$. Si hasta principios de los años noventa las mujeres representaban alrededor del $30 \%$ de los participantes en actividades formativas, en 1996 éstas suponían ya más de la mitad (PSOE, 1997: 96). La debilidad financiera de IU ha impedido la ejecución de programas formativos, a pesar de la voluntad declarada en las sucesivas asambleas (IU, 2003: 13). En el caso del PP no se ha puesto un énfasis especial en la formación política de sus afiliadas.

No obstante, entre las diferencias en la acción positiva, la más visible reside en el poder orgánico que las mujeres han conseguido en los partidos de izquierda en general, y en el PSOE en particular. EI PCE contaba ya en su organización durante la etapa de la clandestinidad con una sección integrada exclusivamente por mujeres, la Comisión de la Mujer. Desde la creación de IU, el Área de Mujer ha sido la sección tradicionalmente encargada del debate de las iniciativas y propuestas políticas que afectan a temas de mujer y de las relaciones con las organizaciones de mujeres. Entre los temas que el Área de Mujer (Área de Movimiento Feminista desde 2004) tiene encomendados estatutariamente se incluyen explícitamente la participación de la mujer y la potenciación de la política feminista en la organización (IU, 2004b: art. 12). No ha habido voluntad por parte de IU de crear organizaciones afines de mujeres, aunque la participación de militantes en las asociaciones de mujeres y la de las mujeres feministas en el partido haya sido un objetivo primordial (IU, 1992: 50).

En el PSOE, la Comisión «Mujer y Socialismo», dependiente de la Secretaría Federal de Formación, se creó en enero de 1977 a instancia de un grupo de militantes del partido que también formaban parte del movimiento feminista autónomo, especialmente del Frente de Liberación de la Mujer ${ }^{4}$. La Secretaría de Participación de la Mujer, creada finalmente en

\footnotetext{
3 Isabel Martínez, ex Coordinadora de la Secretaría de Participación de la Mujer del PSOE (1997-2004), entrevista personal, $18 / 11 / 2004$.

4 La Comisión nunca tuvo una participación masiva, incluso contó en sus inicios con la animadversión de no pocas compañeras de partido que criticaban la autodiscriminación de las mujeres (Fernández Felgueroso, 1990: 196). Tampoco se logró en los primeros años un apoyo especial por parte de los dirigentes del partido (Astelarra, 2005: 134).
} 
septiembre de 1985, se integró hasta 1990 en la Secretaría de Movimientos Sociales y Participación Ciudadana, año en que se constituirá finalmente en Secretaría de Área Federal, consiguiendo así un rango mucho mayor en la estructura orgánica del partido (desde 2000, Secretaría de Igualdad). Para la consecución de sus objetivos, la Secretaría buscó la profundización de las relaciones con el movimiento feminista, el desarrollo de la organización de las mujeres y la promoción de la participación de las militantes socialistas en estas asociaciones, para hacer frente tanto a la tradicional hegemonía conservadora en este sector como a movimientos feministas más radicales y opuestos a la participación institucional que no mostraban ningún apoyo a las políticas para la mujer diseñadas desde el Gobierno del PSOE ${ }^{5}$. Este apoyo a la organización de las mujeres se plasmó en 1987 en la creación de la Federación de Mujeres Progresistas (FMP), que desarrollará un constante trabajo de presión sobre el partido para la participación compartida en el poder ${ }^{6}$. Por otro lado, la Secretaría de Mujer del PSOE ha contado con el apoyo de la Internacional Socialista de Mujeres, organización fraternal de la Internacional Socialista donde participan las secretarías de mujer de los partidos socialistas adscritos a esta última, que ha jugado un rol destacado en la promoción de la cuota en estos partidos desde 1986.

Sin embargo, el PP, a pesar de contar desde 1976 - Alianza Popular, entonces - con un departamento encargado de los temas de mujer, ha rehusado que las mujeres del partido "se especialicen en sus reivindicaciones ${ }^{7}$, mostrando de forma más o menos explícita su rechazo a la creación de un lobby de mujeres en el seno del partido. En los años ochenta se trataba de una vicesecretaría nacional de «promoción femenina» y, a partir de 1989, se constituye en secretaría dentro del Área de Política Social (Secretaría de Igualdad de Oportunidades y Educación desde 1999). No obstante, hasta finales de los años noventa, las relaciones con las organizaciones de mujeres y la elaboración de las políticas de mujer estaban bajo la responsabilidad del Grupo Parlamentario Popular en el Congreso de los Diputados. La inclusión de la Secretaría en el Área de Política Social indica que la mujer se ubica dentro del apartado de «bienestar social» pero no tiene espacio propio; los temas de género se mezclan con los dedicados a la familia, a los menores o a los discapacitados ${ }^{8}$. Este hecho puede interpretarse como el resultado de un todavía débil protagonismo que las mujeres como grupo, que no de forma individual, han tenido en el PP. La literatura ha sostenido que es necesario que exista una «masa crítica» de mujeres antes de que puedan desarrollar una identidad de grupo

5 M. ㄹ Dolors Renau, ex Secretaria de Participación de la Mujer del PSOE en ese momento, entrevista personal, 7/1/2005.

6 Entrevista personal con Enriqueta Chicano, Presidenta de la FMP, 4/11/2004.

7 Declaraciones de José M. ${ }^{\text {a }}$ Aznar en la convención «La mujer en la España de las oportunidades», celebrada por primera vez con motivo del Día de la Mujer (El País, 7 de marzo 1999).

8 Este fenómeno también ha sido observado en los programas electorales (Verge, 2005: 227). 
(Threlfall, 1990: 223). El PP ya dispone hace tiempo de esa masa crítica y el resultado no ha sido el que la teoría podría predecir. Habría que considerar, pues, otros factores como el apoyo de la dirección a la existencia de un lobby de mujeres en su seno.

Las mujeres populares también han contado con el apoyo de una organización de mujeres a la hora de introducir sus demandas en el partido. Sin embargo, su promotor fue Manuel Fraga, quien, en 1982, fundó Mujeres Conservadoras (Mujeres para la Democracia, MD, desde 1993) como respuesta al asociacionismo de mujeres mayormente vinculado a la izquierda. Sus objetivos mezclaban la labor asistencial y la formación con la captación del electorado femenino. Aunque se considera ideológicamente afín al PP, proclama su independencia respecto al partido (Baón, 2001: 829). No obstante, su sede se encuentra en la calle Génova de Madrid, en el mismo edificio que alberga a diferentes grupos de estudio del PP. La influencia de MD sobre las políticas de género del PP ha sido facilitada por la cercanía física de sus sedes y por la doble militancia de muchas mujeres (Ruiz Jiménez, 2002: 354). MD también ha presionado para la inclusión de un mayor número de mujeres en las listas electorales populares ${ }^{9}$.

\section{Estrategias de discriminación positiva}

En cuanto a la discriminación positiva, se han analizado las modificaciones introducidas en los estatutos de los partidos para apoyar una mayor presencia de las mujeres en los cargos orgánicos y públicos.

En 1988, el PSOE introdujo la cuota femenina del 25\% y un año más tarde le seguiría IU, aunque ésta ya había sido aprobada en el PCE en 1987. En 1990, IU elevó el porcentaje al $35 \%$ y, en 1994, el PSOE garantizaba que cuando en un ámbito territorial la afiliación femenina fuera mayor del $25 \%$ la reserva de puestos la superaría en cinco puntos. Las cuotas tardaron unos años en ser respetadas, tanto en los órganos internos como en las listas electorales (especialmente en los puestos iniciales), pero las Secretarías de Mujer no cesaron en su empeño y finalmente consiguieron instaurar la paridad: en 1997, ambos partidos elevan la cuota a un mínimo del $40 \%$ y un máximo del $60 \%$ para cualquier género.

En esos años, las Secretarías de Mujer contaron con el apoyo de organizaciones internacionales como las Naciones Unidas, el Consejo de Europa o la Unión Europea, que instaban a los Estados miembros y a los partidos políticos a adoptar medidas legislativas para hacer efectiva la igualdad. También encontraron el respaldo de organizaciones feministas nacionales ubicadas en la izquierda del espectro político, como la Federación de Mujeres

\footnotetext{
9 Elena Bustillo, Presidenta de la Federación Nacional de MD, entrevista personal, 7/4/2005.
} 
Progresistas, el Forum de Política Feminista o la Coordinadora Española para el Lobby Europeo de Mujeres ${ }^{10}$, asociaciones que se han mostrado muy activas tanto en el ámbito nacional como internacional, organizando numerosos debates sobre la presencia de las mujeres en la toma de decisiones y apoyando diversas campañas a favor de la paridad en las listas electorales (Jenson y Valiente, 2000: 100). Así, las mujeres de los partidos de izquierda podían presentar nuevos avales para exigir la paridad en su partido.

Por el contrario, la discriminación positiva no se ha articulado en el pensamiento político del PP. La máxima del partido es «aquí se está porque se vale, no por cuota sino por nota y porque se tiene la confianza del partido y de la sociedad» (PP, 2002: 148). Además, sostiene la organización que el criterio de elección debe estar basado en las capacidades personales, en el mérito individual, porque, de no ser así, se estaría produciendo una discriminación negativa hacia los hombres ${ }^{11}$. Para el PP, el contenido de las políticas no puede verse afectado por las características de los representantes, en este caso el género, ya que «una Cámara no tiene que ser el reflejo sociológico de una nación»12, sino que el único criterio que debe atenderse es el del mérito y la capacidad. En palabras de Eduardo Zaplana, Portavoz del Grupo Parlamentario Popular en el Congreso de los Diputados: «Los partidos, al confeccionar las listas, tendrán en cuenta la capacidad, competencia y dedicación de cada candidato, y la asunción por el mismo de los principios éticos y políticos que defiendan éstos, quedando excluida toda discriminación por razón de nacimiento, raza, sexo, orientación sexual, edad, religión, discapacidad o cualquier otra condición o circunstancia personal o social (...). Establecer o imponer cualquier tipo de techo para lograr la efectiva igualdad de oportunidades, no supone otra cosa que minusvalorar las capacidades individuales y personales de todo ciudadano ${ }^{13}$.

El PP ha entendido que con la cuota sólo se hace «marketing político» y recuerda que, sin haber implementado ninguna reserva de puestos, fue el Gobierno de Aznar quien designó a mujeres en áreas anteriormente copadas por hombres (por ejemplo, Ana Palacio fue Ministra de

10 En 1993 se organiza en España la «Asociación de Apoyo al Lobby Europeo de Mujeres», impulsada por mujeres de diferentes asociaciones de ámbito estatal a las que, posteriormente, se han ido sumando nuevas organizaciones con el fin de constituir la Coordinadora Estatal para el Lobby Europeo de Mujeres. CELEM se convertía en la homóloga española del Lobby Europeo de Mujeres, creado en Bruselas el 22 de septiembre de 1990 por el impulso de ONGs de los países de la Unión Europea.

11 Juan Carlos Vera, Secretario de Organización del PP, entrevista personal, 31/1/2005.

12 Eugenio Nasarre, ex Secretario de Formación del PP, “Las "listas cremallera" y la democracia representativa», El País, 28 de junio de 2002.

13 Éstos fueron los argumentos utilizados por el PP para rechazar la Proposición de Ley presentada por el Parlamento canario el 16 de abril de 2004 y debatida por el Pleno del Congreso de los Diputados el 2 de noviembre de 2004 (Boletín Oficial de las Cortes Generales, B 5-1, 16 de abril de 2004). Esta propuesta ya había sido presentada en febrero de 2004 (BOCD, D-662, 17 de febrero de 2004). 
Exteriores, y Margarita Mariscal de Gante de Justicia) o que fueron también mujeres las que ocuparon las Presidencias del Congreso y del Senado, o la Vicepresidencia de la Unión Europea (PP, 1996: 67). Desde la Secretaría de Igualdad de Oportunidades la cuota paritaria es contestada justamente desde esta óptica: «La paridad en el Gobierno nos ha demostrado a lo que conduce. ¿Cuántas Secretarias de Estado o Directoras Generales hay? ¿Hablamos de política de cara a la galería o de políticas reales? (...). El PP ha incrementado la participación de las mujeres de forma muy importante sin haber necesitado una política de cuotas ${ }^{14}$.

Igualmente es una opinión unánime entre las mujeres populares que la aplicación de la cuota supone un agravio para las mujeres: «Las mujeres del PP no queremos estar en unas listas electorales por cuotas ni por cremalleras, porque nos parece humillante, porque nos parece un atentando a nuestra dignidad y porque creemos que estamos aquí porque en su día nuestro partido pensó que éramos las mejores»15. Teófila Martínez, ex Presidenta del PP en Andalucía, manifestó que un tratamiento de este tipo equivale a ver a las mujeres como «animales en vías de extinción, susceptibles de ser protegidas». Por su parte, Isabel Tocino, ex Ministra de Medio Ambiente (1996-1999), respaldaba esta misma posición: «Sigo considerando que el que nos admitan como mujeres-cuota no deja de ser una cierta discriminación (...). ¿No seguiremos estando así en corralitos aparte?» ${ }^{16}$.

El rechazo a las cuotas se vio igualmente reflejado en su actividad legislativa en el período 1996-2004. El PP impidió la introducción de la cuota en los sistemas electorales estatal y autonómico. Por un lado, Castilla-La Mancha y Baleares introdujeron en junio de 2002 en sus respectivas leyes electorales la paridad en las listas (50\% de candidatos de cada uno de los dos sexos). El Gobierno del PP interpuso, en septiembre de ese mismo año, sendos recursos de inconstitucionalidad que todavía no han sido resueltos. Por otro lado, en abril de 2002 se presentaron en el Congreso de los Diputados tres Proposiciones de Ley del PSOE, de IU y de Iniciativa per Catalunya-Els Verds para reformar la Ley Orgánica del Régimen Electoral General de 1985 (LOREG) en aras a equilibrar la presencia de hombres y mujeres en las listas electorales. Éstas contaron con el apoyo de toda la oposición y el rechazo del PP17.

14 Sandra Moneo, Secretaria de Igualdad de Oportunidades y Educación del PP, entrevista personal, 25/4/2005.

15 María Mercedes Roldós, diputada del PP, Diario de Sesiones del Congreso de los Diputados, n.ํ 45, 2 de noviembre de 2004.

16 El País, 31 de agosto de 1998.

17 Proposición de Ley del PSOE, Boletín Oficial del Congreso de los Diputados (BOCD) de 16 de noviembre de 2001, n.․171-1; Proposición de Ley de IU, BOCD de 25 de enero de 2002, n.ำ 268-1, y Proposición de Ley de IC-V, BOCD de 31 de julio de 2002, n.ำ 171-1. En años anteriores estos mismos Grupos Parlamentarios habían presentado Proposiciones no de Ley que instaban al incremento de la presencia de las mujeres en los cargos de responsabilidad y decisión política. La propuesta de Izquierda Unida-Iniciativa per Catalunya reclamaba la participación paritaria de hombres y mujeres en los cargos públicos y caducó sin llegar a tramitarse (BOCD, D-24, 14 de junio 1996). Igual suerte corrió la propuesta del PSOE que contenía un plan de acción para la participación de las mujeres en la toma de decisiones (BOCD, D-467, 20 de julio 1999). 


\section{REPRESENTACIÓN DE LA MUJER EN LOS PARTIDOS}

Tras haber analizado las distintas estrategias queremos ver cómo ha evolucionado la presencia de las mujeres en las direcciones ejecutivas de los partidos analizados. ¿Cuál ha sido el efecto que las cuotas han tenido sobre los partidos que las han aplicado? En primer lugar, hay que constatar que las mujeres han ido ganando espacio en los órganos de dirección de todos los partidos de forma progresiva y que, incluso en el PP, a pesar de no haber implementado ninguna medida de discriminación positiva, su presencia se ha triplicado en los últimos veinte años (véase la tabla 1).

TABLA 1

Participación femenina en la Ejecutiva del PP (\%), 1979-2004*

\begin{tabular}{llllllllllllll} 
& 1979 & 1981 & 1982 & 1984 & 1986 & 1987 & 1989 & 1990 & 1993 & 1996 & 1999 & 2002 & 2004 \\
\hline JDN & - & - & - & - & - & - & - & - & 11 & 14 & 15 & N.d. & N.d. \\
\hline CEN & 12 & 4 & 11 & 8 & 13 & 7 & 10 & 18 & 18 & 12 & 22 & 20 & 28
\end{tabular}

* En las siguientes tres tablas, la primera fila se refiere al máximo órgano del partido entre congresos, y la segunda al órgano de tamaño más reducido encargado de aplicar y dirigir la política del partido.

FUENTE:

1979-1999, en Ruiz Jiménez (2002); 2002-2004, datos facilitados por el PP. N.d.: no disponible.

El porcentaje de mujeres en el Comité Ejecutivo Nacional (CEN) del PP en los años ochenta no fue nunca superior al $13 \%$, con proporciones variables e incluso inferiores a este porcentaje (véase tabla 1). A principios de los años noventa se produce un incremento gradual que culmina en 1999 con un $22 \%$ de mujeres en ese órgano y con el porcentaje más alto de mujeres hasta el momento en la Junta Directiva Nacional (JDN), un 15\%. En el XV Congreso (2004) se aprecia la voluntad de seguir incrementando la presencia femenina y las mujeres constituyen ya el $28 \%$ de los miembros del CEN, porcentaje, no obstante, todavía inferior al de las afiliadas populares (33,6\%). En el órgano ejecutivo más operativo, el Comité de Dirección ${ }^{18}$, la presencia de las mujeres es muy minoritaria $(9,1 \%)$.

Respecto al PSOE, en el mismo Congreso en que el partido aprobó la cuota del 25\% (1988) el incremento fue notable tanto en el Comité Federal (CF) como en la Comisión Ejecutiva Federal (CEF): como se observa en la tabla 2, la representación femenina pasó del

18 Lo conforman el Presidente, el Secretario General, los Vicesecretarios Generales, los Secretarios Ejecutivos y los Portavoces del Congreso, del Senado y del Parlamento Europeo. 
5,5 al $13,9 \%$ y del 17,6 al $26,1 \%$, respectivamente. El siguiente impulso a la cuota, adoptado en 1994, produce un nuevo aumento de las mujeres en estos dos órganos y, tras la aprobación de la paridad en 1997, las mujeres superan siempre el 40\%. En 2004 se produce prácticamente un equilibrio de géneros, con un 45,5\% de representación femenina en el CF y del 48,4\% en la CEF. En la actual Comisión Permanente, el órgano de dirección más reducido, las mujeres incluso superan a los hombres $(53,8 \%)$.

TABLA 2

Participación femenina en la Ejecutiva del PSOE (\%), 1981-2004

\begin{tabular}{lllllllll} 
& 1981 & 1984 & 1988 & 1990 & 1994 & 1997 & 2000 & 2004 \\
\hline CF & N.d. & 5,5 & 13,9 & 20,5 & 28,2 & 40,8 & 40,8 & 45,5 \\
\hline CEF & 12,0 & 17,6 & 26,1 & 21,2 & 31,4 & 42,4 & 40,0 & 48,4
\end{tabular}

FUENTE:

Elaboración propia a partir de PSOE (1985, 1998 y 2001). Datos de 2004, en www.psoe.es. N.d.: no disponible.

En cuanto a la aplicación de la cuota en IU, se constata también un incremento gradual de mujeres en la Ejecutiva de la organización, aunque no se ha llegado al cumplimiento efectivo de las previsiones estatutarias (véase tabla 3). Actualmente, las mujeres constituyen el $35 \%$ del Consejo Político Federal (CPF) y el 24,8\% de la Presidencia Ejecutiva Federal (PEF). En la Comisión Permanente el porcentaje de mujeres se ha mantenido estable en las dos últimas Asambleas Federales, alrededor del 32-33\%. Se comprueba, pues, que en los máximos órganos federales de IU existe todavía un «techo de cristal».

TABLA 3

Participación femenina en la Ejecutiva de IU (\%), 1989-2004

\begin{tabular}{lllllllll} 
& 198919 & 1990 & 1992 & 1994 & 1997 & 2000 & 2003 & 2004 \\
\hline CPF & N.d. & N.d. & 28,9 & 30,9 & 29,9 & N.d. & 35,4 & 35,0 \\
\hline PEF & N.d. & N.d. & 25,0 & 25,9 & 29,2 & 21,4 & 28,9 & 24,8
\end{tabular}

FUENTE:

Elaboración propia a partir de los datos facilitados por el Área de Mujer de IU. N.d.: no disponible.

19 A pesar de no disponer de datos sobre la composición de los órganos federales en la primera Asamblea Federal, podemos intuir que la presencia femenina era escasa. La reiterada denuncia del Área de Mujer en los documentos de las sucesivas Asambleas nos hace suponer que su participación no alcanzaba ni siquiera el $25 \%$ de los puestos reservados. 
Son varios los factores que podrían explicar el mayor éxito de la cuota en el PSOE que en IU. En primer lugar, la insistencia en la formación de las mujeres socialistas puede haber facilitado su incorporación en la Ejecutiva al desactivar el argumento del mérito defendido por algunos compañeros de partido. Por otro lado, la existencia de varias corrientes en IU que concurren a las elecciones internas hace que el número de mujeres se vea reducido, ya que los primeros puestos de las listas suelen estar copados, en general, por hombres. Es decir, a mayor competición entre candidatos, constatamos una menor presencia de las mujeres en dichos órganos, en línea con el trade-off presentado por Rahat y Hazan (2005) entre inclusividad y competencia en el interior de los partidos. Por ejemplo, la mayor competición en la presentación de candidaturas para la Coordinación General afecta de forma negativa a la presencia de las mujeres, ya que la configuración de órganos de consenso que integren a miembros de todas las candidaturas termina por primar la presencia de los hombres. Así, hemos visto el dramático descenso de las mujeres en la Comisión Permanente tras la VII (2003) y la VIII Asamblea Federal (2004) al 33 y $32 \%$, respectivamente, cuando concurrieron tres candidatos, frente al $47 \%$ de la VI Asamblea Federal (2000), cuando compitieron sólo dos listas alternativas.

Otras explicaciones podrían apuntar a que esta disposición ha permeado en mayor medida en los distintos niveles territoriales del PSOE que en IU. Así, se observa que entre los representantes elegidos en la Asamblea Federal para el Consejo Político Federal de IU siempre hay más mujeres que entre los designados por las federaciones. En la VIII Asamblea Federal (2004) los porcentajes fueron del $43 \%$ de mujeres en los candidatos designados por la Asamblea, frente al $28 \%$ entre los representantes nombrados por las federaciones. Es decir, el respeto a la cuota es más efectivo en el nivel federal. En cambio, desde las distintas direcciones federales del PSOE se ha desarrollado un constante trabajo de sensibilización en su respeto dirigido a los ámbitos territoriales inferiores ${ }^{20}$. Asimismo, pueden invalidarse aquellas listas que no cumplan con estas medidas y varias mujeres que ocupan altos cargos en la estructura del partido participan en la Comisión Federal de Listas desde 1999. En IU no hay representación de la Secretaría o del Área de Mujer en dicha Comisión²1. Por último, la

$20 \mathrm{El}$ apoyo a las cuotas ha sido constante por parte de los tres últimos Secretarios Generales del PSOE. Felipe González, en el discurso de clausura del XXXII Congreso, manifestó: «El 33\% de las mujeres las he propuesto para que estén representadas en esta Comisión Ejecutiva. (...) Haced este esfuerzo en toda la organización, hacedlo en las listas. No será gratuito, pues eso formará parte, con anticipación, de uno de los grandes cambios que se van a producir en nuestra sociedad» (PSOE, 1994: 246). Joaquín Almunia, el 13 de noviembre de 1999, en el VI Encuentro sobre el Programa Electoral Socialista, declaraba: «Hombres y mujeres tenemos que compartir el poder. La democracia hoy, no es comprensible si no la compartimos hombres y mujeres. Hoy, la democracia tiene que ser una democracia paritaria». Por último, en una entrevista realizada por El Socialista (n. 655, abril de 2004) tras formar Gobierno en abril de 2004, J. L. Rodríguez Zapatero afirmaba que el gobierno paritario «significa un mensaje a toda la sociedad sobre la necesidad de caminar en esa dirección en todos los ámbitos».

21 El CPF aprobó una resolución el 28 de enero de 1995, a instancias de la Secretaría y el Área de Mujer, por la que las direcciones regionales deberían rechazar las candidaturas en las que algún sexo superara el 65\%. Pero en la reunión de la PEF celebrada el 6 de marzo de ese año se modificó la resolución por una invitación a las federaciones a reflexionar sobre la conveniencia de que de cada tres puestos uno estuviera ocupado por una mujer (El País, 7 de marzo de 1995). 
escasa disponibilidad de cargos públicos desde 1999 puede haber convertido los cargos orgánicos en un bien más preciado para los hombres de la formación roji-verde.

\section{REPRESENTACIÓN DE LA MUJER EN LAS INSTITUCIONES}

En cuanto a la evolución de las mujeres en las instituciones, analizaremos en primer lugar la proporción de candidatas que los partidos han venido incorporando en las candidaturas proclamadas para el Congreso, el Senado y el Parlamento Europeo, dado que la inclusión en las listas constituye el filtro previo para los cargos públicos. En segundo lugar, estudiaremos las posiciones que las mujeres han ocupado en dichas listas y, finalmente, examinaremos su efectiva representación institucional.

Respecto al Congreso de los Diputados, hasta 1989 había más mujeres populares candidatas en las listas electorales que socialistas, pero a partir de ese momento el impacto de la cuota aprobada en 1988 es evidente (véase tabla 4). De 1986 a 1989 la presencia de las mujeres en las listas del PP avanza en algo más de dos puntos porcentuales, mientras que en el PSOE se incrementa más de un 200\%. También es en 1989 cuando hay una incorporación masiva de mujeres en las listas de IU, triplicando prácticamente el número de candidatas. EI PCE e IU han sido los partidos que más mujeres han incorporado a sus listas a lo largo del período estudiado, aunque se ven superados por el PSOE a partir del año 2000.

El efecto de las cuotas sobre la representación parlamentaria de las mujeres no fue automático. En las elecciones de 1989 y 1993, con una proporción de mujeres en las listas bastante inferior a la de los partidos de izquierda, el porcentaje de diputadas del PP no estaba tan alejado del que se observaba en los otros dos partidos. En esos años, el PP colocaba a más mujeres como cabezas de lista o en los primeros puestos de la lista que el PSOE. IU superaba en este aspecto al PP, pero la desproporcionalidad del sistema electoral, a pesar de que sus resultados electorales mejoraban, ha jugado en contra de las mujeres de IU. A partir de 1996, el PP se ve ampliamente superado por los partidos de izquierda en todos los aspectos (porcentaje de mujeres en las listas, en la primera mitad de las mismas y en puesto seguro), a excepción de las cabezas de lista, donde las proporciones son muy similares en todos los partidos. Por otro lado, en todos los partidos comprobamos que las mujeres han ocupado tradicionalmente puestos menos adelantados en las listas. Su presencia como cabezas de lista ha sido testimonial hasta 2004, cuando ni siquiera alcanzan entonces el tercio del total de candidaturas.

Desde la introducción de la cuota, las candidatas socialistas en puestos seguros aventajan a las candidatas del PP, sobre todo desde 1996, año en que la competitividad en las elec- 
Porcentaje* y número de mujeres en el Congreso de los Diputados, 1977-2004

\begin{tabular}{|c|c|c|c|c|c|c|c|c|c|}
\hline & $77 / 79$ & $79 / 82$ & $82 / 86$ & $86 / 89$ & $89 / 93$ & $93 / 96$ & $96 / 00$ & $00 / 04$ & $04 / 08$ \\
\hline PSOE & 6,8 & 5,0 & 6,9 & 7,1 & 17,1 & 17,6 & 27,7 & 36,8 & 46,3 \\
\hline Diputadas & (8) & (6) & $(14)$ & $(13)$ & $(30)$ & $(28)$ & (39) & $(46)$ & $(75)$ \\
\hline Porcentaje Congreso & 50,0 & 28,6 & 82,0 & 56,5 & 64,7 & 50,9 & 39,8 & 34,8 & 59,5 \\
\hline Porcentaje lista & 10,3 & 10,2 & 8,3 & 12,5 & 26,8 & 28,9 & 38,1 & 46,4 & 44,1 \\
\hline$(1 / 2-R)$ & 37,2 & 18,7 & 28,8 & 17,4 & 19,7 & 21,6 & 35,5 & 37,2 & 42,1 \\
\hline$(1 / 2-F)$ & 47,2 & 22,0 & 31,5 & 25,8 & 39,0 & 40,6 & 53,2 & 54,7 & 60,3 \\
\hline Puesseg & - & 2,3 & 1,4 & 2,9 & 16,2 & 14,9 & 35,2 & 32,2 & 33,0 \\
\hline Cabeza & 0,0 & 0,0 & 0,0 & 3,8 & 5,9 & 7,7 & 15,4 & 17,3 & 23,1 \\
\hline $\mathrm{PP}^{22}$ & 6,3 & 11,1 & 0,9 & 5,9 & 10,4 & 14,9 & 14,3 & 25,1 & 28,4 \\
\hline Diputadas & (1) & (1) & (1) & (5) & $(11)$ & $(21)$ & (22) & $(46)$ & $(42)$ \\
\hline Porcentaje Congreso & 4,6 & 4,8 & 9,0 & 34,7 & 21,6 & 38,2 & 22,4 & 34,8 & 33,3 \\
\hline Porcentaje lista & 11,5 & 15,1 & 9,9 & 13,0 & 15,3 & 19,3 & 23,0 & 30,9 & 35,8 \\
\hline$(1 / 2-R)$ & 29,5 & 22,8 & 21,4 & 30,1 & 21,4 & 31,8 & 25,7 & 31,9 & 38,3 \\
\hline$(1 / 2-F)$ & 51,5 & 39,7 & 32,1 & 26,5 & 30,7 & 39,3 & 36,4 & 41,7 & 45,6 \\
\hline Puesseg & - & 8,3 & 14,3 & 8,4 & 7,5 & 11,3 & 11,6 & 13,7 & 28,4 \\
\hline Cabeza & 3,8 & 1,9 & 1,9 & 3,8 & 11,5 & 9,6 & 13,5 & 15,4 & 26,9 \\
\hline PCE-IU** & 15,8 & 8,7 & 0,0 & 0,0 & 11,0 & 22,0 & 33,0 & 25,0 & 40,0 \\
\hline Diputadas & (3) & (2) & $(0)$ & $(0)$ & (2) & $(4)$ & $(7)$ & (2) & (2) \\
\hline Porcentaje Congreso & 13,6 & 9,5 & 0,0 & 0,0 & 3,9 & 7,3 & 7,1 & 1,5 & 1,6 \\
\hline Porcentaje lista & 12,2 & 11,8 & 12,1 & 10,4 & 27,8 & 31,5 & 39,2 & 41,7 & 41,1 \\
\hline$(1 / 2-R)$ & 28,0 & 26,2 & 30,2 & 51,8 & 40,5 & 39,2 & 34,4 & 32,0 & 42,6 \\
\hline$(1 / 2-F)$ & 39,7 & 43,5 & 52,6 & 46,3 & 56,3 & 56,9 & 55,5 & 52,6 & 56,6 \\
\hline Puesseg & - & 11,4 & 1,2 & 0,0 & 0,0 & 13,9 & 22,6 & 25,0 & 29,9 \\
\hline Cabeza & 7,7 & 7,7 & 3,8 & 7,7 & 9,6 & 9,6 & 9,6 & 15,4 & 28,8 \\
\hline Diputadas Congreso & 6,0 & 5,0 & 5,9 & 8,4 & 13,9 & 16,0 & 24,0 & 28,3 & 36,0 \\
\hline
\end{tabular}

* Al constituirse la Cámara.

** PCE de 1977 a 1982 e IU de 1986 a 2004.

Diputadas: porcentaje de diputadas en el Grupo Parlamentario.

Porcentaje Congreso: porcentaje de diputadas de cada partido sobre el total de diputadas del Congreso.

Porcentaje lista: porcentaje de mujeres sobre el total de candidaturas proclamadas.

$(1 / 2-R)$ : porcentaje de mujeres en la primera mitad de lista sobre el total de mujeres que incluye cada lista con criterio restrictivo: cuando el número de escaños por provincia es una fracción, se le resta medio punto. Si en una circunscripción la mitad de la lista corresponde al puesto 3.5, sólo se cuentan aquellas mujeres ubicadas hasta el tercer puesto. Por el contrario, en el caso de (1/2-F), un criterio más flexible, se tendrían en cuenta los cuatro primeros puestos de la lista.

Puesseg: porcentaje de mujeres en puesto seguro sobre el total de mujeres de la lista, calculado a partir de los resultados electorales obtenidos por cada partido en las diferentes circunscripciones en la anterior convocatoria electoral.

Cablista: porcentaje de mujeres que ocupa la primera posición de la lista sobre el total de candidaturas proclamadas.

FUENTE:

Elaboración propia a partir de las candidaturas proclamadas: BOE n.ำ 30 (3 febrero 1979), n.ำ 236 (2 octubre 1982), n.ำ 121 (21

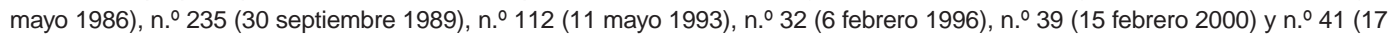
febrero de 2004). Resultados electorales procedentes de fuentes del Ministerio del Interior: www.mir.es.

22 Siglas con las que ha concurrido el PP en las distintas elecciones: Coalición Democrática, Coalición Popular, Alianza Popular y Partido Popular. 
ciones generales ha sido mayor (medida por la incertidumbre del resultado y por el porcentaje de diferencia entre los votos válidos recibidos por los dos principales competidores). Tanto la aprobación de la cuota paritaria en 1997 como su efectivo cumplimiento en la elaboración de las listas de cara a las elecciones generales de 2004 se producen cuando se encuentra en la oposición: el PSOE no podía exigir la reforma de la LOREG en su programa electoral al tiempo que incumplía la paridad en sus propias listas. También es en 1997 cuando IU introduce la paridad en las listas tras haber visto fracasar su objetivo de acercarse o incluso adelantar al PSOE, quedándose lejos de sus expectativas electorales.

Por su parte, en 1993, el PP prácticamente dobló su representación femenina en el Congreso. No hay que olvidar que en estas elecciones se presentaba ya como alternativa de gobierno y que todos sus esfuerzos se dirigían a captar a un sector del electorado socialista. Además, el partido había perdido su ventaja comparativa en el voto femenino respecto a los partidos de izquierda (García Escribano y Frutos, 1999). En el año 2000 se produce un nuevo incremento de las mujeres en el Grupo Parlamentario Popular. El PP no podía seguir acusando al PSOE y a IU de hacer «marketing político» con la cuota si en sus filas la infrarrepresentación de las mujeres era evidente. De este modo, confirmamos que el grado de competitividad de unas elecciones favorece la representación femenina.

En cuanto a la ubicación de las candidatas en las listas, observamos discrepancias notables en todos los partidos si calculamos el porcentaje de mujeres ubicadas en la primera mitad de la lista con un criterio más flexible (1/2-F, en la tabla 4$)$ o más restrictivo (1/2-R). Con el segundo de ellos, el porcentaje de candidatas incluidas en la primera mitad de la lista es sistemáticamente inferior en casi todas las elecciones, siendo la diferencia entre ambas fórmulas superior en los partidos que han aplicado la cuota.

En el Senado se ha producido una evolución similar. Hasta la implantación de la cuota, como podemos observar en la tabla 5, no había muchas discrepancias entre los partidos en relación a la presencia de las mujeres en las listas. EI PCE aventajaba ligeramente al PP y éste al PSOE, pero los porcentajes eran muy bajos en los tres partidos. A partir de 1989 observamos un incremento sustancial en todos los partidos, aunque en el caso del PP este aumento será ralentizado en las sucesivas elecciones, mientras que en los partidos de izquierda seguirá creciendo progresivamente. En 1989, el porcentaje de mujeres en el conjunto de candidaturas era muy similar en los tres partidos: $19 \%$ en el PSOE, $13 \%$ en el PP y $24 \%$ en IU. En 1996 había aumentado al $36 \%$ en los partidos de izquierda y sólo al $17 \%$ en el caso del PP. Sin embargo, en las dos últimas elecciones generales las diferencias se han visto reducidas.

En la elección de senadores/as, el PP ha sido superado tradicionalmente por los partidos de izquierda en la ubicación de mujeres en el primer puesto de la lista. Solamente en las 


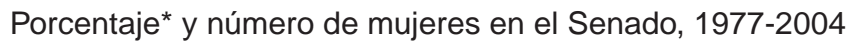

\begin{tabular}{|c|c|c|c|c|c|c|c|c|c|}
\hline & $77 / 79$ & $79 / 82$ & $82 / 86$ & $86 / 89$ & $89 / 93$ & $93 / 96$ & $96 / 00$ & $00 / 04$ & $04 / 08$ \\
\hline PSOE & 3,0 & 3,3 & 6,4 & 8,1 & 14,1 & 17,9 & 22,9 & 17,4 & 27,1 \\
\hline Senadoras & (1) & (2) & (10) & (12) & (18) & (21) & (22) & (12) & (26) \\
\hline Porcentaje Senado & 20,0 & 33,3 & 83,3 & 85,7 & 52,9 & 56,7 & 51,2 & 16,4 & 40,0 \\
\hline Porcentaje lista & 2 & 3 & 6 & 7 & 19 & 25 & 36 & 37 & 39 \\
\hline Cabeza lista & 3,6 & 1,7 & 1,7 & 3,4 & 11,9 & 8,5 & 13,6 & 8,5 & 16,9 \\
\hline Número 2 & 0,0 & 3,4 & 5,1 & 3,4 & 18,5 & 20,3 & 32,2 & 50,8 & 28,8 \\
\hline Número 3 & 0,0 & 3,4 & 8,5 & 10,2 & 16,9 & 33,9 & 37,3 & 11,9 & 37,3 \\
\hline Listas $\sin \mathrm{M}$ & 96,4 & 91,5 & 84,7 & 83,1 & 52,5 & 37,6 & 16,9 & 28,8 & 16,9 \\
\hline PP & 0,0 & 0,0 & 1,47 & 3,1 & 7,8 & 9,4 & 10,5 & 30,9 & 24,6 \\
\hline Senadoras & $(0)$ & $(0)$ & (1) & (2) & (7) & (19) & (14) & (46) & (31) \\
\hline Porcentaje Senado & 0,0 & 0,0 & 8,3 & 14,3 & 20,6 & 27,0 & 32,6 & 63,0 & 47,7 \\
\hline Porcentaje lista & 2 & 4 & 4 & 7 & 13 & 14 & 17 & 34 & 32 \\
\hline Cabeza lista & 1,8 & 3,4 & 5,1 & 3,4 & 6,8 & 3,4 & 5,1 & 13,6 & 25,4 \\
\hline Número 2 & 1,8 & 3,4 & 3,4 & 5,1 & 11,9 & 15,3 & 11,9 & 30,5 & 10,2 \\
\hline Número 3 & 1,8 & 1,7 & 3,4 & 8,5 & 15,3 & 16,9 & 16,9 & 33,9 & 33,9 \\
\hline Listas $\sin \mathrm{M}$ & 94,6 & 91,5 & 88,1 & 83,1 & 66,1 & 64,4 & 66,1 & 22,0 & 30,5 \\
\hline PCE-IU & - & - & 0,0 & 0,0 & 25,0 & 25,0 & 0,0 & 25,0 & 50,0 \\
\hline Senadoras & & & (0) & $(0)$ & (1) & (1) & (0) & (1) & (1) \\
\hline Porcentaje Senado & - & - & 0,0 & 0,0 & 2,9 & 2,7 & 0,0 & 1,4 & 1,5 \\
\hline Porcentaje lista & 6 & 7 & 11 & 13 & 24 & 32 & 36 & 26 & 34 \\
\hline Cabeza lista & 1,8 & 3,4 & 11,9 & 5,1 & 18,6 & 15,3 & 20,3 & 25,4 & 22,2 \\
\hline Número 2 & 1,8 & 5,1 & 6,8 & 11,9 & 11,9 & 22,0 & 33,9 & 10,2 & 35,6 \\
\hline Número 3 & 0,0 & 6,8 & 5,1 & 10,2 & 25,4 & 35,6 & 28,8 & 8,5 & 20,3 \\
\hline Listas $\sin \mathrm{M}$ & 96,4 & 84,7 & 76,3 & 72,9 & 44,1 & 27,1 & 16,9 & 55,9 & 22,0 \\
\hline Total senadoras & 2,32 & 2,32 & 4,35 & 4,9 & 11,6 & 12,4 & 14,0 & 23,0 & 25,1 \\
\hline
\end{tabular}

* Al constituirse la Cámara.

Senadoras: porcentaje de senadoras en el Grupo Parlamentario.

Porcentaje Senado: porcentaje de senadoras de cada partido sobre el total de mujeres en el Senado.

Porcentaje lista: porcentaje de mujeres sobre el total de candidaturas proclamadas.

Cablista: porcentaje de mujeres que ocupa la primera posición sobre el total de candidaturas proclamadas.

Número 2 y Número 3: porcentaje de mujeres ubicadas en la segunda y tercera posición, respectivamente.

Listas $\sin M$ : porcentaje de candidaturas proclamadas sin ninguna mujer.

FUENTE:

Elaboración propia a partir de las candidaturas proclamadas: BOE n. 30 (3 febrero 1979), n.․ 236 (2 octubre 1982), n.ำ121 (21 mayo 1986), n. 235 (30 septiembre 1989), n. 112 (11 mayo 1993), n. 32 (6 febrero 1996), n. 39 (15 febrero 2000$)$ y n. 041 (17 febrero de 2004). Resultados electorales procedentes de fuentes del Ministerio del Interior: www.mir.es. 
elecciones del año 2000 el PP supera al PSOE en mujeres cabeza de lista, y en 2004 a los dos partidos de izquierda. Por otro lado, también hay que poner de manifiesto que, a excepción del año 2000, el PP ha sido el partido que ha presentado más listas sin mujeres. El 17 y el $22 \%$ de las listas del PSOE y de IU en 2004 , respectivamente, no contenían ninguna mujer, porcentajes bastante inferiores al 30,5\% del total de listas populares. De forma progresiva, en los tres partidos, las mujeres han ido escalando puestos en las candidaturas. Aunque en este caso los candidatos no tienen un orden determinado ya que las listas pueden ser alteradas por el votante, en la práctica, el resultado manifiesta una alta similitud con el orden inicial presentado (Montabes y Ortega, 2002). Es decir, los partidos forzosamente conocen que la ubicación en la lista determina las posibilidades de ser elegido.

En el Parlamento Europeo es donde encontramos menores diferencias entre los tres partidos. A pesar de confirmar de nuevo el efecto de las cuotas introducidas en 1989 y 1997 en IU y en el PSOE, vemos en la tabla 6 que la presencia de mujeres en las listas, con la excepción de las elecciones generales de 2004, ha sido mayor para los tres partidos en las candidaturas europeas que en las nacionales. Así, en las elecciones europeas de 1999 y en las generales de 2000 el PSOE presentaba un 46,4\% de mujeres en las listas para el Congreso, un $37 \%$ en las del Senado y un $52 \%$ en las listas del Parlamento Europeo. La misma lógica se aplicaba en el PP (31\% en el Congreso, 34\% en el Senado y $45 \%$ en el Parlamento Europeo) y en IU (41,9\% en el Congreso, $26 \%$ en el Senado y $42 \%$ en el Parlamento Europeo). Por otro lado, el PP ha superado en varias elecciones a los otros dos partidos en número de mujeres situadas en la primera mitad de la lista, pero se ve adelantado por el PSOE en número de mujeres en puestos seguros.

La comparación entre las diferentes elecciones pone de manifiesto que el sistema electoral, si bien no es determinante para explicar la presencia de las mujeres, contribuye a que la representación de las mujeres sea más o menos elevada. Muchos son los autores que han señalado que los sistemas electorales proporcionales con distritos multinominales hacen más fácil la incorporación de los diferentes grupos sociales en las listas electorales (Farrell, 2001: 166; Phillips, 1995: 59; Rule, 1994: 20; Young, 2000: 152). Por otro lado, cuanto mayor sea la magnitud del distrito, más mujeres tienen posibilidades de resultar elegidas (Norris y Franklin, 1997). En el cuadro 1 se resumen las características de los distintos sistemas electorales empleados en España.

Al analizar longitudinalmente la composición de las distintas instituciones comprobamos que la representación de las mujeres ha sido tradicionalmente más alta en el Parlamento Europeo que en el Congreso, y mayor en el Congreso que en el Senado. Esto es, cuanto más proporcional sea el sistema electoral y cuantos más representantes se elijan en las circunscripciones, más mujeres obtienen representación. A su vez, un mal resultado elec- 
Porcentaje y número de mujeres en el Parlamento Europeo, 1987-2004

\begin{tabular}{|c|c|c|c|c|c|}
\hline & 1987 & 1989 & 1994 & $1999^{23}$ & $2004^{24}$ \\
\hline \multicolumn{6}{|l|}{ PSOE } \\
\hline Diputadas & $11(3)$ & $19(5)$ & $36(8)$ & $42(10)$ & $44(11)$ \\
\hline Porcentaje lista & $12(7)$ & $33(20)$ & $47(30)$ & $52(33)$ & $46(25)$ \\
\hline Porcentaje (1/2) & $43(3)$ & $25(5)$ & $43(13)$ & $45(15)$ & $44(11)$ \\
\hline Puesto seguro & -* & $25(5)$ & $30(9)$ & 27 (9) & $40(10)$ \\
\hline \multicolumn{6}{|l|}{ PP } \\
\hline Diputadas & $6(1)$ & $7(1)$ & $29(8)$ & $37(10)$ & $29(7)$ \\
\hline Porcentaje lista & $17(10)$ & $12(7)$ & $25(16)$ & $45(29)$ & $33(18)$ \\
\hline Porcentaje (1/2) & $30(3)$ & $52(4)$ & $50(8)$ & $41(12)$ & $38(7)$ \\
\hline Puesto seguro & -* & $14(1)$ & $31(5)$ & $34(10)$ & $39(7)$ \\
\hline \multicolumn{6}{|l|}{ IU } \\
\hline Diputadas & $0(0)$ & $25(1)$ & $44(4)$ & $25(1)$ & $0(0)$ \\
\hline Porcentaje lista & $12(7)$ & $35(21)$ & $48(31)$ & $42(27)$ & $46(25)$ \\
\hline Porcentaje (1/2) & $43(3)$ & $43(9)$ & $39(12)$ & $59(16)$ & $40(10)$ \\
\hline Puesto seguro & - & $0(0)$ & $6(2)$ & $11(3)$ & $8(2)$ \\
\hline Total delegación española & $10(6)$ & $15(9)$ & $33(21)$ & $34(22)$ & $33(18)$ \\
\hline
\end{tabular}

* Los anteriores representantes españoles habían sido elegidos por designación.

Porcentaje lista: porcentaje de mujeres sobre el total de candidaturas proclamadas.

Porcentaje (1/2): porcentaje de mujeres en la primera mitad de lista sobre el total de mujeres de cada lista.

Puesseg: porcentaje de mujeres en puesto seguro sobre el total de mujeres de la lista, calculado a partir de los resultados electorales obtenidos por cada partido en las diferentes circunscripciones en la anterior convocatoria electoral.

FUENTE:

Elaboración propia a partir de las candidaturas proclamadas: BOE n.ำ108 (6 mayo de 1987), n.ำ110 (9 mayo de 1989 ),

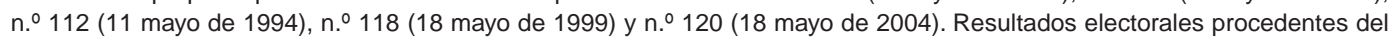
Ministerio del Interior: www.mir.es.

\section{CUADRO 1}

\section{Características de los sistemas electorales en España}

\begin{tabular}{lllcc} 
Elecciones & \multicolumn{1}{c}{ Sistema electoral } & Tipo de listas & $\begin{array}{c}\text { Número } \\
\text { de distritos }\end{array}$ & $\begin{array}{c}\text { Magnitud } \\
\text { media }\end{array}$ \\
\hline Congreso & $\begin{array}{l}\text { Sistema proporcional } \\
\text { (corregido) Ley d'Hont }\end{array}$ & Cerradas y bloqueadas & 52 & 6,7 \\
\hline Senado & $\begin{array}{l}\text { Sistema mayoritario } \\
\text { multinominal - Voto limitado }\end{array}$ & Panachage & 60 & 4 \\
\hline Parlamento Europeo & Sistema proporcional puro & Cerradas y bloqueadas & 1 & 54
\end{tabular}

23 En estas elecciones, Loyola de Palacio (PP) y Rosa Díez (PSOE) concurrieron como cabezas de lista. Fue la primera convocatoria electoral europea en que una mujer ocupaba la primera posición.

24 Se ha calculado la proporción que representaría el nuevo puesto seguro al reducirse los escaños de 64 a 54 en las elecciones europeas de 2004: PSOE, 28\%; PP, 33\%; IU, 4\%. 
toral para un partido tiende a penalizar mucho más a las mujeres en los sistemas mayoritarios, ya que, en comparación con los hombres, ellas ocupan en menor medida el primer y el segundo puesto en la lista, como también indican los datos ofrecidos en la tabla 5. Analizando en profundidad las elecciones al Senado de 2004 se observa que las listas no bloqueadas son neutras respecto a la preferencia por las mujeres del electorado. De las 60 circunscripciones provinciales, sólo se produjeron alteraciones en las listas en 4 de ellas. En cuanto a las mujeres se refiere, tenemos alteraciones en sólo 3 casos: en 2 de ellos la mujer desplaza al hombre y en el tercero es el hombre quien desplaza a la mujer. Tanto por el escaso número de circunscripciones en que este fenómeno sucede como por su bidireccionalidad, no se puede concluir que las listas no cerradas favorezcan a la mujer, sino más bien afirmar que, en líneas generales, los votantes siguen la lista del partido sin hacer uso de la posibilidad de distribuir de forma diferente a los candidatos. Así, dado que los sistemas mayoritarios favorecen menos la representación de la mujer, que los electores no modifican el orden presentado por los partidos y que los partidos ubican a un escaso porcentaje de mujeres en el primer puesto, el resultado es una presencia femenina mucho menor en el Senado que en el resto de instituciones.

En 2004 resultaron elegidas un 36\% de mujeres en el Congreso de los Diputados, un $33,3 \%$ en el Parlamento Europeo y un $25,1 \%$ en el Senado. En este último caso, la magnitud del distrito no nos explica por qué hay menos mujeres en el Parlamento Europeo que en el Congreso de los Diputados, siendo ambos sistemas proporcionales y el utilizado en las elecciones europeas mucho más proporcional debido a la magnitud de la circunscripción (todo el territorio nacional). La clave está en las estrategias de los partidos. Por un lado, la aplicación efectiva de la cuota por parte del $\operatorname{PSOE}^{25} \mathrm{y}$, por otro lado, el fracaso electoral del PP en las elecciones generales de 2004. En 2004, a pesar de que el porcentaje de mujeres populares en el Congreso ha aumentado, han disminuido las parlamentarias europeas de este partido. La pérdida de 35 escaños en las últimas elecciones generales y del Gobierno (y, en consecuencia, de la capacidad de nombrar altos cargos) convirtieron los puestos en el Parlamento Europeo en un bien mucho más codiciado, lo que supuso un ligero retroceso de la presencia institucional de las mujeres populares ${ }^{26}$.

\footnotetext{
25 También en IU, pero de nuevo los resultados electorales son determinantes. De hecho, el porcentaje de hombres en puesto seguro en el caso de IU sería bastante parecido al de las mujeres.

26 De los 24 europarlamentarios del PP electos en 2004, 17 son hombres. Entre éstos, seis ocuparon altos cargos bajo el Gobierno de Aznar: dos ex Ministros (Jaime Mayor Oreja y Cristóbal Montoro); el Portavoz del Grupo Parlamentario Popular en el Congreso durante la VI y la VII Legislaturas, Luis de Grandes; dos Directores Generales, y un asesor personal de Aznar. Además, los ex dirigentes regionales del partido en Cataluña y el País Vasco, Aleix Vidal-Quadras y Carlos Iturgaiz, se encuentran también entre los nuevos parlamentarios europeos. Todos ellos representan un tercio de la delegación popular española. El PSOE también utilizó las listas para el Parlamento Europeo de 1999 para reservar algunos puestos a ex cargos del Gobierno o ex miembros de la Ejecutiva previa al XXXIV Congreso (1997), pero el porcentaje de mujeres no disminuyó; al contrario, se logró sobrepasar la cuota paritaria.
} 
Finalmente, cabe analizar la representación de las mujeres en el poder ejecutivo. La primera constatación es que ésta es la instancia en que el dominio masculino es más evidente, aunque se observa que cada nuevo Gobierno ha igualado o aumentado el número de mujeres (véase tabla 7). A nivel ministerial es donde la presencia de la mujer más se ha visto incrementada, pasando de una ministra en 1982 al Gobierno paritario constituido en abril de 2004, tras dos décadas con un número de ministras que oscilaba entre 3 y $5^{27}$. En cuanto a los niveles inferiores, subsecretarías y direcciones generales, la participación de las mujeres ha seguido una tendencia creciente desde los años ochenta, pese a que la tasa de incremento ha sido muy baja. Es también en estos niveles de menor responsabilidad y/o visibilidad pública donde la infrarrepresentación de las mujeres es menos acentuada. Por último, en cuanto a las secretarías de Estado, la representación femenina en 2002 era incluso inferior a la de 1995. Su reducción se inició en 1996 y se profundizó en los últimos ocho años siguientes hasta experimentar un nuevo crecimiento en 2004.

TABLA 7

Porcentaje y número de mujeres en los altos cargos del Gobierno

\begin{tabular}{|c|c|c|c|c|c|c|c|c|c|c|}
\hline & $\begin{array}{r}1982 \\
\text { (UCD) }\end{array}$ & $1986^{*}$ & 1989 & 1993 & $\begin{array}{r}\text { Marzo } \\
1995\end{array}$ & $\begin{array}{r}\text { Mayo } \\
1996\end{array}$ & $\begin{array}{l}\text { Sep. } \\
1998\end{array}$ & $\begin{array}{r}\text { Oct. } \\
2000\end{array}$ & $\begin{array}{l}\text { Sep. } \\
2002\end{array}$ & $\begin{array}{l}\text { Nov. } \\
2004\end{array}$ \\
\hline Ministerios & $\begin{array}{l}5,6 \\
(1)\end{array}$ & $(0)$ & (2) & $\begin{array}{r}11,1 \\
(3)\end{array}$ & $\begin{array}{r}20,0 \\
(3)\end{array}$ & $\begin{array}{r}20,0 \\
(3)\end{array}$ & $\begin{array}{r}28,6 \\
(4)\end{array}$ & $\begin{array}{r}18,8 \\
(3)\end{array}$ & $\begin{array}{r}20,0 \\
(3)\end{array}$ & $\begin{array}{r}50,0 \\
(8)\end{array}$ \\
\hline Secretarías de Estado & $\begin{array}{r}0,0 \\
(0)\end{array}$ & (1) & - & 0,0 & $\begin{array}{r}25,0 \\
(5)\end{array}$ & $\begin{array}{r}21,7 \\
(5)\end{array}$ & $\begin{array}{r}4,2 \\
(1)\end{array}$ & $\begin{array}{r}3,9 \\
(1)\end{array}$ & $\begin{array}{r}3,5 \\
(1)\end{array}$ & $\begin{array}{r}12,0 \\
(3)\end{array}$ \\
\hline Subsecretarías & $\begin{array}{l}0,0 \\
(0)\end{array}$ & (1) & (1) & 5,3 & $\begin{array}{l}9,1 \\
(2)\end{array}$ & $\begin{array}{r}8,1 \\
(3)\end{array}$ & $\begin{array}{l}6,8 \\
(4)\end{array}$ & $\begin{array}{l}19,1 \\
(13)\end{array}$ & $\begin{array}{r}25,4 \\
(17)\end{array}$ & $\begin{array}{r}22,2 \\
(16)\end{array}$ \\
\hline Direcciones generales & $\begin{array}{l}1,4 \\
(3)\end{array}$ & (23) & (23) & 13,7 & $\begin{array}{l}13,4 \\
(37)\end{array}$ & $\begin{array}{l}14,5 \\
(37)\end{array}$ & $\begin{array}{l}13,2 \\
(27)\end{array}$ & $\begin{array}{r}16,0 \\
(34)\end{array}$ & $\begin{array}{l}17,1 \\
(36)\end{array}$ & $\begin{array}{r}22,9 \\
(51)\end{array}$ \\
\hline Total (\%) & 1,4 & & & 12,8 & 14,4 & 11,4 & 11,8 & 16,1 & 17,2 & 21,7 \\
\hline
\end{tabular}

* Sólo se dispone de los datos absolutos de mujeres de 1986 y 1989, sin poder calcular el porcentaje que representan. En 1993 sólo se dispone de los datos porcentuales.

Fuente:

Elaboración propia a partir de los datos facilitados por la Subdirección General de Organización del Ministerio de Administraciones Públicas; 1982-93, en Instituto de la Mujer (1999), y 1986-89, en Sánchez Hernández (2003: 161).

27 De 1982 a 1986 no hubo ninguna ministra en el Gobierno. De 1988 a 1991 hubo dos ministras, y de 1993 a 1995, tres. En el primer Gobierno del PP (1996-2000) empezaron cuatro ministras y terminaron dos. En el segundo mandato popular (20002004) empezaron tres ministras y terminaron cinco (Instituto de la Mujer, 2004: Mujeres en cifras, www.mtas.es/mujer). 


\section{MÁS ALLÁ DE LAS CUOTAS: LIMITACIONES Y RETOS}

En estos veintiséis años de democracia en España, la mujer se ha incorporando progresivamente a las instituciones y a los puestos de responsabilidad en los partidos. En paralelo a esta evolución discurren otros fenómenos que dificultan un equilibrio de género real y efectivo en el ejercicio del poder político, tales como una renovación más alta de mujeres que de hombres, la segmentación de las responsabilidades políticas o los constreñimientos derivados de la compaginación de la vida familiar con la política.

Tanto en los tres partidos aquí estudiados como en el conjunto del Congreso, las mujeres acumulan menos experiencia política ya que aproximadamente el $60 \%$ de las mismas sólo ha permanecido una legislatura en el Congreso, frente al $52,5 \%$ de los hombres. En la tabla 8 observamos que el grado de permanencia en número de legislaturas en todos los partidos analizados y en el conjunto de partidos del arco parlamentario es mayor en el caso de los hombres. Si tenemos en cuenta las personas que han ostentado el cargo durante sólo una legislatura, el porcentaje siempre es superior en las diputadas: $66 \%$ frente a $50 \%$ de los hombres en el PSOE, $60 \%$ frente a $39 \%$ en IU, $60 \%$ frente a $54 \%$ en el PP, y $63 \%$ frente a $53 \%$ en la media del Congreso. Curiosamente, es en los partidos que han adoptado la cuota donde la renovación de las mujeres es mayor.

TABLA 8

Permanencia de los/as diputados/as (\%)

\begin{tabular}{|c|c|c|c|c|c|c|c|c|}
\hline \multirow[b]{2}{*}{ Número de legislaturas } & \multicolumn{3}{|c|}{ PSOE } & \multicolumn{2}{|l|}{ PP } & IU & \multicolumn{2}{|c|}{ Media Congreso } \\
\hline & $\mathrm{H}$ & M & $\mathrm{H}$ & M & $\mathrm{H}$ & M & $\mathrm{H}$ & M \\
\hline Una & 50,3 & 65,6 & 53,9 & 60,0 & 38,7 & 60,0 & 52,5 & 62,7 \\
\hline Dos & 20,1 & 17,5 & 20,0 & 21,7 & 35,5 & 26,7 & 23,0 & 20,6 \\
\hline Tres & 10,2 & 9,3 & 11,5 & 12,2 & 19,4 & 13,3 & 10,3 & 10,2 \\
\hline Cuatro & 6,7 & 3,8 & 8,9 & 2,6 & 4,8 & 0 & 6,4 & 2,8 \\
\hline Cinco & 5,8 & 2,2 & 3,3 & 2,6 & 1,6 & 0 & 4,0 & 2,5 \\
\hline Seis & 3,3 & 1,6 & 1,7 & 0,9 & 0 & 0 & 2,1 & 1,1 \\
\hline Siete & 1,8 & 0 & 0,4 & 0 & 0 & 0 & 1,0 & 0 \\
\hline Ocho & 1,8 & 0 & 0,2 & 0 & 0 & 0 & 0,8 & 0 \\
\hline
\end{tabular}

FUENTE:

Elaboración propia a partir del archivo de diputados facilitado por el Archivo del Congreso de los Diputados.

Si se analiza legislatura a legislatura y por partidos, la renovación de las mujeres es también casi siempre mucho mayor que la de los hombres en IU y en el PSOE que en el PP, partido que muestra la tendencia inversa (véase tabla 9). Como hemos visto, la preocupa- 
TABLA 9

Permanencia por legislaturas (porcentaje y número)

\begin{tabular}{|c|c|c|c|c|c|c|}
\hline & \multicolumn{3}{|c|}{ PSOE } & \multicolumn{2}{|l|}{ PP } & IU \\
\hline & $\mathrm{H}$ & M & $\mathrm{H}$ & M & $\mathrm{H}$ & M \\
\hline Legislatura Constituyente (1977/79) & $62,7(74)$ & $44,4(4)$ & $22,2(4)$ & $100(1)$ & $84,2(16)$ & $33,3(1)$ \\
\hline I Legislatura (1979/82) & $62,2(84)$ & $75,0(6)$ & $64,3(9)$ & $100(1)$ & $16,7(4)$ & $0,0(0)$ \\
\hline II Legislatura (1982/86) & $60,1(125)$ & $52,6(10)$ & $37,4(40)$ & $25,0(1)$ & $0,0(0)$ & $0,0(0)$ \\
\hline III Legislatura (1986/89) & $64,7(119)$ & $73,7(14)$ & $43,8(46)$ & $46,2(6)$ & $37,5(3)$ & $0,0(0)$ \\
\hline IV Legislatura (1989/93) & $65,6(103)$ & $56,8(21)$ & $60,6(66)$ & $75,0(9)$ & $62,5(10)$ & $50,0(1)$ \\
\hline V Legislatura (1993/96) & $47,7(71)$ & $39,4(13)$ & $67,6(94)$ & $73,9(17)$ & $47,1(8)$ & $100(4)$ \\
\hline VI Legislatura (1996/00) & $43,6(48)$ & $46,8(22)$ & $61,0(94)$ & $67,7(21)$ & $31,3(5)$ & $16,7(1)$ \\
\hline VII Legislatura (2000/04) & $48,2(41)$ & $44,6(25)$ & $49,1(78)$ & $34,8(23)$ & $14,3(1)$ & $0,0(0)$ \\
\hline Media por género 1977/2004 & 56,8 & 54,2 & 50,7 & 65,3 & 36,7 & 25,0 \\
\hline
\end{tabular}

FUENTE:

Elaboración propia a partir de los datos facilitados por el Archivo del Congreso.

ción creciente del PP por incorporar a más mujeres entre sus cargos habría favorecido este hecho: la mayoría de las diputadas son mantenidas en las listas. Ahora bien, en la última legislatura sólo el 35\% de las mujeres populares, frente al $49 \%$ de los hombres de su partido, han renovado su acta. Se produce en el PP, a partir del año 2004, un fenómeno observado en el PSOE desde 1993: a medida que aumenta la presencia de las mujeres en el Grupo Parlamentario, éstas sufren sistemáticamente una mayor renovación que los hombres. De nuevo, la ubicación en las listas es fundamental: a peor resultado electoral, más sufren sus consecuencias los candidatos que no ocupan puestos de salida. En IU, a excepción del período 1989-1996, la renovación es, en general, muy alta, especialmente entre las mujeres.

En otras ocasiones, la asunción de la cuota ha significado un incremento o una consolidación de áreas políticas asociadas tradicionalmente a la mujer que segregan por sexos la actividad política (Diz Otero y Lois González, 2004: 218). Veamos en primer lugar qué sucede en el interior de los partidos. En la Comisión Ejecutiva Federal del PSOE hay ocho Secretarias Ejecutivas y ocho Secretarias con «cartera»: Relaciones Internacionales, Igualdad, Educación y Ciencia, Política Económica y Empleo, Bienestar Social, Cultura, Medio Ambiente y Desarrollo Rural. En la Presidencia Ejecutiva Federal de IU encontramos nueve Secretarias en Política Municipal y Autonómica, Libertades, Cultura, Solidaridad y Cooperación, Igualdad, Inmigración, Relaciones Institucionales y Portavoz Institucional. Y, por último, en el Comité Ejecutivo Nacional del PP hay dos Secretarias Ejecutivas en Política Social y Política Autonómica y Local, y tres Secretarias en Libertades Públicas, Seguridad y Justicia, Política Social y Bienestar y Economía y Empleo. Se observa, pues, una 
segmentación horizontal por funciones tanto más pronunciada cuanto más equilibrada sea la presencia de la mujer en el partido. A su vez, las presidencias, las secretarías generales y las secretarías de organización, las áreas de mayor poder orgánico, están en manos, en general, tanto a nivel nacional como en los niveles territoriales inferiores, de hombres.

La segmentación horizontal también se observa por ministerios. Apreciamos en la tabla 10 que los ministerios ocupados por mujeres han tenido principalmente un contenido sociocultural (57\%, ó 64\% si incluimos el Ministerio de Medio Ambiente). Sólo un 14,3\% de las ministras ha tenido bajo su responsabilidad las carteras de funciones básicas, y un $9,5 \%$ las económicas ${ }^{28}$.

TABLA 10

Segmentación horizontal por ministerios 1982 1986 1989 1993 1996 $2000 \quad 2004$ Total

Funciones básicas

Funciones económicas Infraestructura

Funciones socioculturales

Total ministras

$\begin{array}{llll} & 1 & \\ 1 & & 1 & 3 \\ 1 & 0 & 2 & 3\end{array}$

$\begin{array}{lll}1 & & \\ 1 & & \\ 1 & 3 & 3 \\ 1 & 3 & 8\end{array}$

(2004

$\begin{array}{cc}1 & 3(14,3 \%) \\ 1 & 2(9,5 \%) \\ 3 & 4(19,1 \%) \\ 3 & 12(57,1 \%) \\ 8 & 21(100 \%)\end{array}$

FUENTE:

Elaboración propia a partir de Mujeres en cifras (2004). Datos del inicio de la legislatura.

Un último ámbito donde se puede observar esta pauta es en las comisiones parlamentarias $^{29}$. Las mujeres han pasado de ser el $12,5 \%$ de los miembros de las comisiones de más rango (Tipo 1) en 1989 al 34,5\% en 2004, y han aumentado del 20,9 al 45,6\% en las de menor rango (Tipo 2). La presencia siempre ha sido más equilibrada en aquellas comisiones de contenido social (Tipo 3), pasando del 40\% en 1989 al 54,6\% en 2004. Por partidos,

\footnotetext{
28 Funciones básicas: Administraciones Públicas, Defensa, Justicia, Asuntos Exteriores y Cooperación, Interior, Presidencia, Presidencia del Gobierno y Portavocía del Gobierno. Funciones económicas: Economía y Hacienda, Industria, Turismo y Comercio, Agricultura, Pesca y Alimentación, etc. Infraestructura: Fomento, Medio Ambiente y Vivienda. Funciones socioculturales: Trabajo y Asuntos Sociales, Educación y Ciencia, Sanidad y Consumo y Cultura. Esta clasificación está basada en la elaborada por el Instituto de la Mujer, Mujeres en cifras. www.mtas.es/mujer.

29 Seguimos la clasificación establecida por Valiente et al. (2004): Tipo 1: Comisiones permanentes legislativas y Comisiones del Tribunal de Cuentas y de la UE. Tipo 2: Comisiones permanentes no legislativas, Comisiones de estudio y Comisiones mixtas de Relaciones con el Defensor del Pueblo, de Derechos de la Mujer y de Estudio del problema de las drogas. Tipo 3: Comisiones dedicadas a políticas sociales (Educación, Cultura, Deporte, Política Social, Empleo, Sanidad y Consumo, Cooperación Internacional, Mixta de Derechos de la Mujer y Mixta del Problema de las Drogas).
} 
en el intervalo 1989-2004, el PSOE e IU siempre han estado por encima de la media parlamentaria en la participación de las mujeres en las distintas comisiones, a pesar de que la tendencia ha sido similar: más mujeres en las comisiones de Tipo 3 que en las de Tipo 2 y que en las de Tipo 1, sucesivamente. Por el contrario, el PP siempre ha estado por debajo de la media, aunque la presencia de las mujeres populares en las distintas comisiones se haya prácticamente duplicado en este período (véase el Apéndice).

En las distintas comisiones también hay un dominio masculino en los cargos de más rango (Presidencia, Vicepresidencia, Secretaría y Portavocía) y una mayor participación de las mujeres en las Vocalías y Adscripciones. Hay en la actualidad un 26,7\% de Presidentas, un $29,1 \%$ de Vicepresidentas, un $50,9 \%$ de Secretarias, un $36,8 \%$ de Portavoces, un $49,3 \%$ de Portavoces adjuntas, un 38,7\% de Vocales y un 45,9\% de Adscritas. De nuevo se ha observado un incremento notable desde 1989 y una diferenciación por partidos, teniendo el PSOE y IU muchas más mujeres que el PP en cargos de mayor rango. En cuanto a la presencia de las mujeres en la Diputación Permanente del Congreso, el porcentaje se ha elevado del $4 \%$ en la IV Legislatura al $30 \%$ en la VIII. En la Mesa el incremento ha sido del $450 \%$ en este período (1989-2004), pero en la Junta de Portavoces el dominio masculino no ha variado, habiendo sólo aumentado la presencia de las mujeres del 6 al $11 \%$.

En último lugar, existen una serie de recursos y motivaciones que los candidatos traen consigo al proceso de reclutamiento. Según varios autores, estos «factores de oferta», como el capital político, el tiempo disponible o el background ocupacional, resultan altamente determinantes a la hora de optar a un puesto. La política es una profesión altamente demandante en disponibilidad de horarios, de desplazamientos, etc. (Norris y Franklin, 1997: 201). Así, observamos en la VIII Legislatura que el $82,5 \%$ de los diputados están casados, frente al $59,4 \%$ de las diputadas. El siguiente dato resulta igualmente interesante: las ocho ministras del Gobierno de Rodríguez Zapatero suman cinco hijos; los ocho ministros, veintidós.

La modificación de las pautas y dinámicas organizativas ha sido una reclamación permanente de las mujeres socialistas y comunistas desde los años ochenta y una queja constante de las Secretarías de Mujer. EI Plan de Igualdad del PSOE (2002) prevé la prestación de un servicio de guardería para atención de niños y niñas menores de tres años, ofrecido a trabajadores/as y a quienes acuden a reuniones, y la ampliación del permiso por maternidad con un permiso especial por paternidad para los trabajadores del partido, cuando el varón sea quien lo disfrute. No se han concretado, sin embargo, medidas para modificar los horarios de las reuniones. También las mujeres de IU han reclamado reiteradamente la modificación de las formas de participación política en el interior del partido a fin de permitir que se puedan conciliar con las necesidades de desarrollo personal de mujeres y hombres —horarios, multiplicidad de reuniones, etc.- (IU, 2003: 13), pero no se han desarrollado 
hasta la fecha medidas específicas. Por otro lado, el Pleno del Congreso de los Diputados votó unánimemente el 13 de diciembre de 2004 una Proposición no de Ley, consensuada entre el PSOE y CiU, en la que se insta al Ejecutivo a adoptar las medidas que posibiliten compatibilizar el ejercicio de las funciones representativas y el derecho a disfrutar del período de baja por maternidad, adopción o acogimiento, aunque, de momento, no se han iniciado acciones concretas ${ }^{30}$.

Esta opinión es también generalizada en la sociedad española. Según una encuesta realizada en 2002 (CIS, estudio n.․ 2448), el 77\% de las mujeres, frente al $62 \%$ de los hombres, cree que para llegar a puestos directivos en la vida política las mujeres tienen más dificultades que los hombres. Por otro lado, los electorados de IU y del PSOE consideran que la situación de las mujeres en el acceso a la política es mucho peor que los votantes del PP. Mientras que el $72 \%$ de las votantes del PP creen que esto es así, en el PSOE e IU los porcentajes se elevan al 79 y al $84 \%$. En cuanto a los hombres, se observa una tendencia parecida: el 54\% de los votantes varones del PP, el 66\% de los del PSOE y el $69 \%$ de los de IU consideran que las mujeres tienen mayores dificultades para acceder a las responsabilidades políticas. Esta preocupación es, pues, mayor en el electorado de los partidos que han implementado la cuota y que han incrementado de forma notable la representación política de las mujeres.

\section{CONCLUSIONES}

El incremento en la representación femenina se ha producido tanto por cuestiones ambientales derivadas de la competición electoral entre los partidos, como por cuestiones internas, especialmente a partir de la constitución de lobbies de militantes feministas en los partidos de izquierda que, desde los años ochenta, fueron ganando poder orgánico y que, posteriormente, desde la Secretaría de Mujer presionaron a sus compañeros de partido hasta conseguir la introducción estatutaria de la cuota. De hecho, el activismo de las mujeres de los partidos y el establecimiento de cuotas legales se confirman como los factores fundamentales que influyen en la representación política de las mujeres, en consonancia con los datos presentados por otros estudios (Caul, 2005). Sin embargo, resultó igualmente clave el respaldo de la dirección del partido, un apoyo de importancia fundamental para lograr extender su aplicación a toda la organización y vencer algunas resistencias internas.

Por otro lado, las Secretarías pudieron hacer valer en esos años el apoyo que, tanto distintas organizaciones internacionales como organizaciones feministas de ámbito nacional,

30 BOCG, n.․ 58, de 14 de diciembre de 2004. 
prestaban a las medidas de discriminación positiva. No obstante, a pesar del importante papel desempeñado por estas asociaciones, el liderazgo ha correspondido a las militantes y dirigentes de los partidos de izquierda (Jenson y Valiente, 2001: 102).

La ideología política del partido ha sido también determinante en este proceso. Hemos comprobado, en línea con el planteamiento de Lovenduski y Norris (1993), que el compromiso con la representación de las mujeres en la retórica del partido ha sido el inicio de un proceso que ha conducido a políticas de inclusión más amplias en los partidos de izquierda, que se han acompañado de diferentes medidas de acción y de discriminación positiva con efectos notables en la presencia orgánica e institucional de las mujeres. En cambio, la acción y la discriminación positiva no han encontrado su espacio en el pensamiento político popular, contrario a la constitución de lobbies de mujeres en el partido y a la introducción de cuotas.

Aun así, la competición partidista ha actuado como un «círculo virtuoso»: con cada alternancia entre socialistas y populares, el Gobierno entrante ha igualado o incrementado la proporción de mujeres en su gabinete y en cada legislatura el número de mujeres no ha cesado de aumentar hasta lograr que, en la actualidad, España se ubique en el octavo puesto del ranking mundial en representación femenina en las Cámaras legislativas ${ }^{31}$ y que se encuentre entre los pocos países del mundo que cuentan con un Gobierno paritario.

\section{APÉNDICE}

\begin{tabular}{|c|c|c|c|c|c|c|c|c|}
\hline \multirow[t]{2}{*}{ Comisión Tipo 1} & \multicolumn{3}{|c|}{ PSOE } & \multicolumn{2}{|l|}{ PP } & \multirow{2}{*}{$\frac{\text { IU }}{2004}$} & \multicolumn{2}{|c|}{$\begin{array}{r}\text { TOTAL } \\
\text { CONGRESO }\end{array}$} \\
\hline & 1989 & 2004 & 1989 & 2004 & 1989 & & 1989 & 2004 \\
\hline Presidencia & 0,00 & 30,77 & 0,00 & 50,00 & 一 & - & 0,00 & 31,58 \\
\hline Vicepresidencia & 28,57 & 47,06 & 14,29 & 6,67 & 一 & - & 21,43 & 26,47 \\
\hline Secretaría & 50,00 & 36,84 & 6,67 & 43,75 & 一 & - & 27,59 & 40,00 \\
\hline Portavocía & 一 & 32,00 & - & 24,00 & 一 & 35,29 & - & 23,03 \\
\hline Portavocía adjunta & 一 & 46,15 & - & 38,46 & 一 & - & - & 42,31 \\
\hline Vocalía & 17,28 & 43,32 & 8,64 & 23,43 & 6,67 & 0,00 & 11,69 & 33,92 \\
\hline Adscripción & 一 & 53,92 & - & 11,11 & 一 & - & - & 46,21 \\
\hline TOTAL SEXOS & 18,41 & 44,87 & 8,85 & 25,00 & 6,67 & 33,33 & 12,50 & 34,49 \\
\hline
\end{tabular}

31 Datos correspondientes a 31 de julio de 2006. Esta clasificación puede consultarse en la página web de la Unión Interparlamentaria: http://www.ipu.org/wmn-e/classif.htm. 


\begin{tabular}{|c|c|c|c|c|c|c|c|c|}
\hline \multirow[t]{2}{*}{ Comisión Tipo 2} & \multicolumn{3}{|c|}{ PSOE } & \multicolumn{2}{|l|}{ PP } & \multirow{2}{*}{$\frac{\text { IU }}{2004}$} & \multicolumn{2}{|c|}{ CONGRESO } \\
\hline & 1989 & 2004 & 1989 & 2004 & 1989 & & 1989 & 2004 \\
\hline Presidencia & 11,11 & 28,57 & 0,00 & 0,00 & - & - & 9,09 & 18,18 \\
\hline Vicepresidencia & 27,27 & 55,56 & 22,22 & 18,18 & - & - & 23,81 & 33,33 \\
\hline Secretaría & 77,78 & 90,91 & 22,22 & 37,50 & - & 100,00 & 40,91 & 68,18 \\
\hline Portavocía & - & 33,33 & - & 33,33 & - & 77,78 & 一 & 34,72 \\
\hline Portavocía adjunta & - & 87,50 & - & 55,56 & - & - & - & 70,59 \\
\hline Vocalía & 26,32 & 53,28 & 17,28 & 41,28 & 22,22 & 50,00 & 19,76 & 46,69 \\
\hline Adscripción & - & 65,00 & - & 0,00 & - & - & 一 & 50,00 \\
\hline TOTAL SEXOS & 28,18 & 56,45 & 17,82 & 38,93 & 22,22 & 75,00 & 20,89 & 45,58 \\
\hline
\end{tabular}

\begin{tabular}{|c|c|c|c|c|c|c|c|c|}
\hline \multirow[t]{2}{*}{ Comisión Tipo 3} & \multicolumn{3}{|c|}{ PSOE } & \multicolumn{2}{|l|}{ PP } & \multirow{2}{*}{$\frac{\text { IU }}{2004}$} & \multicolumn{2}{|c|}{$\begin{array}{r}\text { TOTAL } \\
\text { CONGRESO }\end{array}$} \\
\hline & 1989 & 2004 & 1989 & 2004 & 1989 & & 1989 & 2004 \\
\hline Presidencia & 33,33 & 71,43 & - & - & - & - & 33,33 & 66,67 \\
\hline Vicepresidencia & 66,67 & 85,71 & 66,67 & 22,22 & 一 & - & 66,67 & 50,00 \\
\hline Secretaría & 66,67 & 66,67 & 33,33 & 44,44 & - & - & 50,00 & 55,56 \\
\hline Portavocía & - & 50,00 & - & 50,00 & - & 77,78 & - & 42,31 \\
\hline Portavocía adjunta & - & 84,62 & - & 61,54 & 一 & - & - & 73,08 \\
\hline Vocalía & 49,25 & 62,75 & 32,26 & 44,19 & 42,86 & - & 38,46 & 54,26 \\
\hline Adscripción & - & 74,36 & - & 0,00 & - & - & - & 63,64 \\
\hline TOTAL SEXOS & 50,00 & 67,20 & 35,14 & 43,94 & 42,86 & 77,78 & 40,00 & 54,62 \\
\hline
\end{tabular}

\begin{tabular}{|c|c|c|c|c|c|c|c|c|}
\hline \multirow[t]{2}{*}{ Total Comisiones } & \multicolumn{3}{|c|}{ PSOE } & \multicolumn{2}{|l|}{ PP } & \multirow{2}{*}{$\frac{\text { IU }}{2004}$} & \multicolumn{2}{|c|}{$\begin{array}{r}\text { TOTAL } \\
\text { CONGRESO }\end{array}$} \\
\hline & 1989 & 2004 & 1989 & 2004 & 1989 & & 1989 & 2004 \\
\hline Presidencia & 4,17 & 30,00 & 0,00 & 25,00 & - & - & 3,70 & 26,67 \\
\hline Vicepresidencia & 28,00 & 50,00 & 17,39 & 11,54 & - & - & 22,45 & 29,09 \\
\hline Secretaría & 60,87 & 56,67 & 12,50 & 41,67 & - & 100,00 & 33,33 & 50,88 \\
\hline Portavocía & - & 32,35 & - & 26,47 & - & 50,00 & 一 & 26,79 \\
\hline Portavocía adjunta & - & 55,88 & - & 42,86 & - & - & 一 & 49,28 \\
\hline Vocalía & 20,52 & 46,90 & 11,52 & 30,28 & 12,50 & 33,33 & 14,55 & 38,75 \\
\hline Adscripción & 26,32 & 52,03 & 17,28 & 40,74 & 22,22 & 50,00 & 19,76 & 45,92 \\
\hline TOTAL SEXOS & 21,98 & 48,43 & 11,95 & 29,98 & 12,50 & 50,00 & 15,55 & 38,20 \\
\hline
\end{tabular}




\section{BIBLIOGRAFÍA}

ASTELARRA, Judith (2005): Veinte años de políticas de igualdad, Valencia: Cátedra.

BAÓN, Rogelio (2001): Historia del Partido Popular. Del franquismo a la refundación, vol. I, Madrid: Ibersaf.

BUDGE, Ian; KLINGEMANN, Hans-Dieter; VOLKENS, Andrea; BARA, Judith, y TANEMBAUM, Erik (2001): Mapping policy preferences. Estimates for parties, electors and governments 1945-1988, Oxford: Oxford University Press.

CAUL, Miki (1999): «Women’s Representation in Parliament: The Role of Political Parties», Party Politics, vol. 5, n. .91 , pp. 79-98.

COORDINADORA ESPAÑOLA PARA EL LOBBY EUROPEO DE MUJERES (1999): Hacia una democracia paritaria: análisis y revisión de las leyes electorales vigentes, Madrid: CELEM.

DIZ OTERO, Isabel, y LOIS FERNÁNDEZ, Marta (2004): «La presencia política de las mujeres. Una comparación de la clase política y la opinión pública gallegas», Zona Abierta, n.ำ106-107, pp. 175-223.

FARRELL, David M. (2001): Electoral systems, Nueva York: Palgrave.

FERNÁNDEZ FELGUEROSO, Paz (1990): «Las propuestas del socialismo y el feminismo sobre la participación política de las mujeres», en Judith Astelarra (ed.), Participación política de las mujeres, Madrid: CIS.

GARCÍA ESCRIBANO, Juan José, y FRUTOS, Lola (1999): «Mujeres, hombres y participación política. Buscando las diferencias», REIS, n. 86 , pp. 307-329.

INSTITUTO DE LA MUJER (1999): La situación de la mujer en la toma de decisiones, Madrid: Instituto de la Mujer.

— (2004): Mujeres en cifras. www.mtas.es/mujer.

IU (1986): Programa Elecciones Generales de 1986: El compromiso de IU con la sociedad española.

— (1992): Resoluciones de la III Asamblea Federal (mayo).

— (2003): Resoluciones de la VII Asamblea Federal (diciembre).

— (2004a): Resoluciones de la VIII Asamblea Federal (diciembre).

— (2004b): Estatutos Federales aprobados en la VIII Asamblea Federal (diciembre).

JENSON, Jane, y VALIENTE, Celia (2001): «El movimiento a favor de la democracia paritaria en Francia y España», Revista Española de Ciencia Política, n.ํㅜ 5, pp. 79-110.

LOVENDUSKI, Joni (1993): «Introduction: the dynamics of gender and party», en Joni Lovenduski y Pippa Norris (eds.), Gender and Party Politics, Thousand Oaks, California: Sage.

- (1997): «Representación política: dinámica de género y partidos», en Edurne Uriarte y Arantxa Elizondo (eds.), Mujeres en política, Barcelona: Ariel.

LOVENDUSKI, Joni, y NORRIS, Pippa (eds.) (1993): Gender and Party Politics, Thousand Oaks, California: Sage.

MONTABES, Juan, y ORTEGA, Carmen (2002): «El voto limitado en las elecciones al Senado español: estrategias de nominación y rendimientos partidistas en las elecciones de marzo de 2000", Revista Española de Ciencia Política, n. $\stackrel{\circ}{7}$, pp. 103-130.

NORRIS, Pippa (1993): “Conclusions: comparing legislative recruitment», en Joni Lovenduski y Pippa Norris (eds.), Gender and Party Politics, Thousand Oaks, California: Sage. 
NORRIS, Pippa, y FRANKLIN, Mark (1997): «Social representation», European Journal of Political Research, n.ำ 32 , pp. 185-210.

PANEBIANCO, Angelo (1990): Modelos de partido. Organización y poder en los partidos políticos, Madrid: Alianza.

PHILLIPS, Anne (1995): The politics of presence, Nueva York: Clarendon Press.

PP (1993): Resoluciones del XI Congreso Nacional (febrero).

- (1996): Resoluciones del XII Congreso Nacional (enero).

- (2002): Un nuevo impulso para España. Resoluciones del XIV Congreso Nacional (enero).

— (2004a): Programa Elecciones Generales de 2004.

- (2004b): Nuestro partido hoy (documento del XV Congreso, octubre).

PSOE (1985): Documento sobre índices de representación de las mujeres socialistas, Madrid: PSOE, Secretaría de Participación de la Mujer.

- (1988): Resoluciones del XXXI Congreso Federal (enero).

- (1993): Programa Elecciones Generales de 1993.

- (1994): Resoluciones del XXXIII Congreso Federal (marzo).

— (1997): Resoluciones del XXXIV Congreso Federal (junio).

- (1998): La participación de las mujeres en el PSOE y en las Instituciones Públicas, Madrid: PSOE, Secretaría de Igualdad.

- (2001): La participación de las mujeres en el PSOE y en las Instituciones Públicas, Madrid: PSOE, Secretaría de Igualdad.

- (2002): I Plan de Igualdad, Madrid: PSOE, Secretaría de Igualdad.

RAHAT, Gideon, y HAZAN, Reuven (2005): «On the difference between democracy within parties and democracy within status: the uneasy relationship between participation, competition and representation», ECPR workshop, Granada, 14-19 de abril.

RAMIRO, Luis (2004): Cambio y adaptación en la izquierda. La evolución del Partido Comunista de España y de Izquierda Unida (1986-2000), Madrid: CIS.

RUIZ JIMÉNEZ, Antonia M. (2002): Mecanismos del cambio ideológico e introducción de políticas de género en partidos conservadores: el caso de AP-PP en España en perspectiva comparada, Madrid: CEACS, Fundación Juan March. Tesis doctoral.

RULE, Wilma (1994): «Parliaments of, by, and for the People: except for women?», en Wilma Rule y Joseph F. Zimmerman (eds.), Electoral systems in comparative perspective. Their impact on women and minorities, Westport, CT: Greenwood Press.

SÁNCHEZ FÉRRIZ, Remedio (2000): «Las mujeres en las Cortes Generales y en los Parlamentos de las Comunidades Autónomas», en AA.VV., Mujer y Constitución en España, Madrid: Centro de Estudios Políticos y Constitucionales.

SÁNCHEZ HERNÁNDEZ, María F. (2003): Liderazgo político de mujeres. Desde la transición hasta las cuotas, SeviIla: Instituto Andaluz de la Mujer. 
THRELFALL, Mónica (1990): «¿Patriarca, palanca, paraguas? Planteamientos feministas en torno al estado asistencial», en Judith Astelarra (ed.), Participación política de las mujeres, Madrid: CIS.

URIARTE, Edurne (1995): «Mujer y política en España», Sistema, n.ำ124, pp. 121-136.

VALIENTE, Celia; RAMIRO, Luis, y MORALES, Laura (2004): «Women in the Spanish Parliament», en Yvonne Galligan y Manon Tremblay (eds.), Sharing power: women, parliament and democracy, Aldershot: Ashgate.

VERGE, Tània (2005): Partidos y representación política: las dimensiones del cambio en los partidos políticos españoles, 1977-2004, Madrid: Instituto Universitario de Investigación Ortega y Gasset - Universidad Complutense. Tesis doctoral.

YOUNG, Iris M. (2000): Inclusion and democracy, Oxford: Oxford University Press. 